\title{
Heuristics versus Norms: On the Relativistic Responses to the Kaufmannn Experiments ${ }^{\text {th }}$
}

\author{
Jan Potters ${ }^{1}$ \\ Centre for Philosophical Psychology, Department of Philosophy, University of Antwerp, \\ Grote Kauwenberg 18, 2000 Antwerpen, Belgium
}

\section{Abstract}

The aim of this article is to provide a historical response to Michel Janssen's (2009) claim that the special theory of relativity establishes that relativistic phenomena are purely kinematical in nature, and that the relativistic study of such phenomena is completely independent of the dynamics of the systems displaying such behavior. This response will be formulated through a historical discussion of one of Janssen's cases, the experiments carried out by Walter Kaufmann on the velocity-dependence of the electron's mass. Through a discussion of the different responses formulated by early adherents of the principle of relativity (Albert Einstein, Max Planck, Hermann Minkowski and Max von Laue) to these experiments, it will be argued that the historical development of the special theory of relativity argues against Janssen's historical

\footnotetext{
The author would like to thank Richard Staley, Hasok Chang, Marco Giovanelli, Harvey Brown, Dennis Lehmkuhl, Thomas Ryckman, and the audiences at the Fifth Young Researchers Day in Logic, History and Philosophy of Science 2016 (Brussels), the Forum Scientiarium Summerschools of 2017 and 2018 (Tübingen), and the EPSA 2017 conference (Exeter). This article was partially written during a stay as a visiting student at the Department of History and Philosophy of Science at the University of Cambridge (UK).

Email address: Jan.Potters@UAntwerpen. be (Jan Potters)

${ }^{1}$ Corresponding Author, Research Funded by the FWO (Research Foundation - Flanders)
} 
presentation of the case, and that this raises questions about his general philosophical claim. It will be shown, more specifically, that Planck and Einstein developed a relativistic response to the Kaufmann experiments on the basis of their study of the dynamics of radiation phenomena, and that this response differed significantly from the response formulated by Minkowski and Laue. In this way, it will be argued that there were, at the time, two different approaches to the theory of relativity, which differed with respect to its relation to theory, experiment, and history: Einstein's and Planck's heuristic approach, and Minkowski's and Laue's normative approach. This indicates that it is difficult to say, historically speaking, that the special theory of relativity establishes the kinematical nature of particular phenomena. Instead, it will be argued that the theory of relativity should not be seen as a theory but rather as outlining an approach to the interpretation of physical theories, and that the nature of particular scientific phenomena is something that is open to scientific debate and dispute.

Keywords: Kaufmann experiments, velocity-dependence of mass, special relativity, kinematics versus dynamics

\section{Introduction}

Recently, Michel Janssen (2009) has argued, by means of three historical cases, that the special theory of relativity establishes that various phenomena, previously thought to require an explanation in terms of the dynamics of the system displaying the behaviour, can in fact be given an entirely kinematical explanation, and that this entails that the relativistic study of these phenomena is completely independent of the system's dynamics. One of these 
cases concerns Walter Kaufmann's experiments on the velocity-dependence of the electron's mass. While Hendrik Lorentz and Max Abraham attempted to account for Kaufmann's results in terms of the dynamics of the electron, special relativity shows, according to Janssen, that the phenomenon is independent of the dynamics underlying the system displaying it, and that it is rather a consequence of the relativistic space-time in which the system displays this behavior. Janssen argues for this claim, more specifically, by means of three historical claims: (i) that Albert Einstein's (1905a) derivation of the relativistic equations describing the velocity-dependence of mass shows that he saw that the phenomenon was purely kinematical; (ii) that the relativistic account of the velocity-dependence of mass was mainly elaborated and accepted on the basis of theoretical considerations; and (iii) that the work by Max von Laue clearly shows the kinematical nature of the phenomenon because it makes use of Hermann Minkowski's space-time geometry, which forms the natural interpretation of the theory of special relativity.

In this article, I will argue that the historical development of the theory of special relativity argues against Janssen's claims (i)-(iii). By means of a discussion of how different adherents of the principle of relativity (Einstein, Max Planck, Minkowski and Laue) responded to the issues raised by Kaufmann's experiments, I will argue, in particular, for the following claims: (i) that both Einstein and Planck did not consider the phenomenon to be purely kinematical in nature, and that dynamical considerations, based on their study of radiation phenomena, did play an important role in the development of their response to Kaufmann's experiments; (ii) that besides theoretical considerations, experimental and historiographical factors also played an important 
role in the establishment of a relativistic account of the experiments; and (iii) that this shows that both Einstein and Planck adhered to a heuristic approach to the theory of relativity that differed profoundly from what I will call Minkowski's normative approach, and that therefore, historically speaking, we should not see the Minkowskian framework as necessarily offering the natural interpretation of the theory of relativity. Through the elaboration of these three points, I will then argue that Laue's work does not completely address the issues raised by Kaufmann's experiments, but rather sidesteps them in certain ways, because of its use of the Minkowskian approach. By contrasting the Minkowskian normative approach with the Einsteinian heuristic approach, I will then conclude that it is difficult to claim that the theory shows that the velocity-dependence of mass is kinematical in nature. The historical discussion rather shows that such characterizations of the nature of a phenomenon are dependent on a particular scientist's aims and interests, and are therefore open to scientific debate and dispute.

In order to achieve this, I will proceed as follows. I start, in section 2, with a short discussion of Janssen's general philosophical claims and his historical discussion of the Kaufmann experiments. After that, I will turn to a discussion of the historical episode (section 3), in which I will focuson Janssen's historical claims (i)-(iii). In section 4, I will then elaborate some concluding remarks.

\section{Janssen's Discussion of the Kaufmann Experiments}

The following statement perfectly summarizes the essential novelty of Einstein's Special theory of Relativity (STR), according to Janssen (2009, p. 
26): "Einstein was the first to formulate clearly the new kinematical foundation for all of physics inherent in Lorentz's electron theory" (Stachel et al., 1989, p. 253). The theory's main accomplishment, on this reading, was that it showed that relativistic phenomena such as time dilation and length contraction are purely kinematical. That a phenomenon is kinematical means, on Janssen's view, that "it is just an instance of some generic feature of the world, in this case instances of default spatio-temporal behavior" (2009, p. 27). Janssen elaborates this claim by means of a distinction between two kinds of kinematical phenomena: phenomena are kinematical in the broad sense if they are "independent of the specifics of the dynamics"; they are kinematical in the narrow sense if they are "an example of standard spatiotemporal behavior" (2009, p. 28). STR now shows that certain phenomena are kinematical in both senses, which entails that nothing more is to be learned from them: "[u]nless one challenges the classification of the phenomenon as kinematical in this sense - and the universality of the relevant feature will militate strongly against that - there is nothing more to learn from that particular phenomenon, neither about the specific system in which it occurs nor about the generic feature it instantiates" (Janssen, 2009, p. 27; original emphasis).

Janssen's argument for this claim consists of three particular historical episodes in which STR established the kinematical nature of particular phenomena previously thought to require a dynamical account ${ }^{2}$ : refraction in

\footnotetext{
${ }^{2}$ These are the specific examples that Janssen presents as arguments for his general claim. His reason for discussing these is that they have not received the philosophical attention they deserve (Janssen, 2009, p. 29) in comparison with e.g. the Michelson-Morley
} 
moving media and the Fresnel drag coefficient (2009, p. 29 - 32); the Kaufmann experiments and the velocity-dependence of mass (2009, p. 32 - 41); and the Trouton-Noble experiment (2009, p. $41-47)$. Each of these cases shows, according to Janssen, how STR explains these phenomena "by identifying the kinematical nature (rather than the cause) ${ }^{3}$ of the relevant phenomena" (2009, p. 28). As such, the best way to clarify and illustrate Janssen's general claim is through a historical and philosophical discussion of one of these cases, namely the Kaufmann episode. The main reason for discussing this episode is that, as Janssen points out, it has not yet received the philosophical attention it deserves, especially since the Kaufmann experiments were central to the debate in the scientific community at the time. ${ }^{4}$ Moreover, while they were taken by Poincaré, Lorentz and others to pose serious issues for the theory of relativity at the time (see (Miller, 1981, p. $334-$ 335) and (Staley, 2008, chapters 6 and 7)), relatively few has been written about the development of a relativistic response. Finally, the discussion of historical episode suggests an interesting point, which is that the elaboration of a relativistic theory really only started in the years after the publication

experiment, which, he claims, also backs up his claim (Janssen, 2009, p. 48).

${ }^{3}$ Such causal interpretations of STR, as offered for example by Harvey Brown in his (2005) book, are the main foil of Janssen's argument. In short, Brown's dynamical account of special relativity comes down to the claim that the relativistic phenomena should not be accounted for in terms space-time geometry, but rather in terms of the dynamics underlying these phenomena.

${ }^{4}$ As we will see, Kaufmann was the first to discuss Einstein's (1905a) relativity article, and it was mainly in the context of the issues raised by the experiments that Einstein's work was elaborated, discussed and criticized (Staley, 2008, p. 242 - 243). 
of Einstein's (1905a). The main reason for discussing Janssen's philosophical claim from a historical perspective is that he himself also favours this approach: he describes his work as "a brand of philosophy of physics informed by (conceptual) history of physics" $(2009$, p. 28$) .^{5}$

Pre-relativistic treatments of the Kaufmann experiments. Between 1901 and 1906, Walter Kaufmann carried out a series of experimental measurements of the exact dependence of the electron's mass on its velocity, with the goal to provide insight into the electron's constitution. Such a dependence was first hinted at by J. J. Thomson, who claimed that if the electron moves through its own electromagnetic field, it should experience a decrease in velocity as if it had gained mass (Staley, 2008, p. 219) (Miller, 1981, p. 46). While Thomson presented this as merely a mathematical hypothesis, the idea gained physical meaning a few years later. The first to elaborate the physical content of this dependence was H. A. Lorentz (1899). In the first part of this article Lorentz presents a new, simplified, formulation of the transformation equations for coordinates and electric and magnetic fields he had proposed in his Versuch (1895). In the second part, he then elaborates some of the physical consequences of the deformation hypothesis he had presented earlier in response to the result of the Michelson-Morley experiment, by considering its implications for a particular example, i.e. an oscillating electron. Determining the specific transformation equations for the forces and accelerations involved in such a system then leads him to suggest that

\footnotetext{
${ }^{5}$ Besides his (2009), Janssen also has an extensive article on this case with Matthew Mecklenburg (2006).
} 
the mass of an electron in motion depends on its velocity. Janssen formulates Lorentz's hypothesis as follows: ${ }^{6}$

It follows that the electron's oscillation in the moving frame only satisfies Newton's second law if its mass depends on its velocity and if its 'longitudinal mass $\left(m_{\|}\right)$, the inertia for acceleration in the direction of motion, differs from its 'transverse mass' $\left(m_{\perp}\right)$, the inertia for acceleration perpendicular to the direction of motion. More specifically, $m_{\|}$and $m_{\perp}$ should satisfy (Lorentz (1899, p. 442); Lorentz (1904, p. 27))

$$
m_{\|}=l \gamma^{3} m^{\prime}, m_{\perp}=l \gamma m^{\prime}
$$

Both $m_{\|}$and $m_{\perp}$ increase dramatically as the velocity of the electron approaches the velocity of light. (Janssen, 2009, p. 33)

Here, $l$ denotes an unspecified scale factor, $\gamma=1 / \sqrt{1-(v / c)^{2}}$, and $m^{\prime}$ refers to the material system's rest mass. Lorentz's electron-theory (1904), which models the electron as"a small spherical surface charge distribution, which undergoes a microscopic version of the Lorentz-Fitzgerald contraction when it is set in motion with respect to the ether", later brought him to put $l=1$ (Janssen, 2009, p. 34). ${ }^{7}$ At around the same time, however, a different formula for the electron's velocity-dependent mass was also proposed by Max Abraham (1902; 1903; 1904; 1905). His account, which modeled the electron

\footnotetext{
${ }^{6}$ See Janssen and Mecklenburg (2006, p. 75 - 80) for a more extensive discussion of how Lorentz arrives at this claim.

${ }^{7}$ In this way, Janssen (2009, p. 32) points out, Lorentz thus arrived at the same equations as STR, even though Einstein obtained them through a different route, as we will see later.
} 
as "a rigid spherical surface charge distribution [which] retains its shape regardless of its state of motion", was in line with his commitment to the electromagnetic program, ${ }^{8}$ since it entailed a complete dependence of the electron's mass on its motion through its self-field, and as such suggested an electromagnetic account of matter (Janssen, 2009, p. 33 - 34). The difference between Lorentz's and Abraham's equations for the electron's longitudinal and transverse mass thus stemmed from their different electron-models - rigid versus deformable - which in turn, as Janssen (2009, p. 34) points out, derived from their different concerns: Lorentz's electron was deformable because he wanted his theory to account for the material contraction of the material set-up used in the Michelson-Morley experiment; ${ }^{9}$ Abraham's electron was

\footnotetext{
${ }^{8}$ In very general terms, the electromagnetic view of nature was an approach to physical theorizing that attempted to reduce the whole of physics to interactions in the electromagnetic ether obeying the Maxwell equations. Its most well-known adherents were Wilhelm Wien, who wrote a kind of manifesto (1900), and Abraham (see e.g. his (1905, p. 143 147) handbook). Recent years have seen the emergence of a debate about the historical meaning of this movement/program, and I therefore refer the reader to those articles for a more elaborate and informed discussion of the electromagnetic view, as well as for an overview of the existing literature on the subject: see the article by Suman Seth (2004) which drew a reply from Shaul Katzir (2005), to which Seth again replied (2005). Chapter 24 of Christa Jungnickel and Russell McCormmach 's history of nineteenth-century theoretical physics $(1990$, p. 211 - 253) offers a thorough discussion of the electromagnetic program and its relations to the main other world views at the time.

${ }^{9}$ To account for the null result of Michelson and Morley's experiments, Lorentz claimed that the length of the interferometer's arms deformed because of their motion through the ether. To explain how this occured, Lorentz hypothesized that the electrons constituting the material set-up were subject to the same deformation as the optical interference patterns measured in the experiment (Janssen\&Mecklenburg, 2006, p. 75 - 79) (Janssen,
} 
rigid because he wanted his theory to be in line with the electromagnetic program.

In this way, the velocity-dependence of the electron's mass offered the possibility to investigate the constitution of the electron: was it rigid or deformable? To investigate this question, Kaufmann carried out a series of experiments (1901a; 1902; 1903; 1906) in which he investigated the charge-tomass ratio of the electrons constituting high-velocity Becquerel rays (which later became known as $\beta$-rays). ${ }^{10}$ He did this by passing these rays, emitted by a piece of radium bromide, through a diaphragm in a lead barrier, onto a photographic plate, all within a flask that was evacuated of air. When no electric or magnetic fields were applied, the electrons that constituted the rays would form a simple dot on the plate. The application of a magnetic field would lead to the image of a straight line, whereas the application of both an electric and a magnetic field would give rise to a curved line. Because of the variation of the velocity of the electrons, this curvature could be taken to represent the velocity-dependence of the electron's charge-to-mass ratio: the higher the electron's velocity, the more it would be deflected by the applied fields. At the time, electrodynamics was taken to imply that an electron's

2009, p. 32).

${ }^{10}$ Unfortunately, I cannot go much into the details of the way in which Kaufmann set up and ran his experiments. James T. Cushing (1981), Arthur Miller (1981, p. 47 - 54; 61 - 67; 226 - 232), Giora Hon (1995) and Richard Staley (2008, p. 219 - 243) all offer extensive discussions of Kaufmann's experimental set-up and his conceptual apparatus. Regarding the material aspects of Kaufmann's experiments specifically, I refer the reader to chapter six of Staley's (2008) book on Einstein's generation and the origins of the relativity revolution. 
charge remains stable with changing velocity (Cushing, 1981, p. 1138). Given the recent experimental determinations of the electron's charge-to-mass ratio by Thomson and Pieter Zeeman, Kaufmann could thus infer a precise formula for the velocity-dependence of the electron's mass from the precise curvature of the curve on the photographic plate.

The first round of experiments showed a definite dependence of the electron's mass on its velocity (Kaufmann, 1901a, p. 155). Abraham (1902, p. 21) soon pointed out, however, that Kaufmann's derivation of his results only allowed him to make that claim for the electron's longitudinal mass $m_{\|}$, but not for what was actually at stake, namely its transverse mass $m_{\perp} \cdot{ }^{11}$ This was a consequence of Kaufmann's reliance on a defective electron-model, proposed by G. F. C. Searle (1897). Abraham therefore developed an alternative model of the electron, i.e. his rigid spherical charge. This in turn prompted Kaufmann to reanalyse his earlier data, and to conduct a new series of experiments. These showed a very close fit with Abraham's predictions, which led Kaufmann to the conclusion that the electron's mass is of a purely electromagnetic nature (Kaufmann, 1902, p. 295).

These results prompted Lorentz to elaborate a purely electromagnetic account of the electron as well, which led him to put $l=1$ in the equations (1) for the electron's velocity-dependence of mass (1904, p. 24 - 31). Abraham ((1904, p. 578); (1905, p. 204 - 205)) soon pointed out, however,

\footnotetext{
${ }^{11}$ In fact, it was in this context that the distinction between longitudinal and transverse mass was made for the first time, by Abraham in his (1902) article. Before, most physicists spoke in more general terms about the distinction between a body's true (mechanical) mass, and its apparent (electrodynamical) mass (Staley, 2008, p. 229 - 230).
} 
that Lorentz's electron, in its purely electromagnetic form, possibly faced stability-issues: its $m_{\|}$derived from its electromagnetic momentum differs from its $m_{\|}$derived from its electromagnetic energy by a factor $4 / 3$, which entailed that Lorentz had to give up the fully electromagnetic nature of his electron: it required "an extra, nonelectromagnetic force in order to retain equilibrium, for without it the deformable electron could explode owing to the repulsive forces between the constituents of its charge" (Staley, 2008, p. 231). ${ }^{12}$. Moreover, in (1903), Abraham had shown that force-free inertial motion, i.e. "inertial motion that could be maintained without any external forces balancing the electron's self-forces", was not guaranteed unless the electron's velocity and momentum were collinear (Miller, 1981, p. 57). This was the case, he showed, when "the charge's shape is symmetric about axes perpendicular to its direction of motion" (Miller, 1981, p. 58). For Abraham's rigid spherical charge, this was of course no problem. When one conceived the electron as a rigid ellipsoidal charge distribution, this entailed that force-free inertial motion was only possible when moving in the direction of its major axis. Finally, for Lorentz's deformable electron, it was not clear how an additional non-electromagnetic force, required for the cohesion of Lorentz's deformable electron, would ensure that force-free inertial motion was possible in all directions (Miller, 1981, p. 76). The solution was presented by Poincaré $((1905$, p. 578); $(1906$, p. 130)): it was only by assuming that the electron experiences a negative pressure from the ether that Lorentz's equations for the electron's longitudinal and transverse mass (with $l=1$ )

\footnotetext{
${ }^{12}$ Miller offers a very extensive discussion of this issue in chapter 1 of his (1981) book, as do Stanley Goldberg (1970) and Janssen \& Mecklenburg (2006, p. 85 - 88).
} 
could be retained (Janssen, 2009, p. 35).

Because Abraham's and Lorentz's models led to different predictions for the velocity-dependence of mass, Kaufmann (1906) decided to run another series of experiments, which were carried out with enormous attention to detail and accuracy (Staley, 2008, p. 236 - 241). Abraham's and Lorentz's were not the only theories tested, however. Another electron model that was considered was one proposed by Alfred Bucherer (1904). Bucherer's electron was deformable, just as Lorentz's, but the way in which it deformed in motion differed in such a way that its volume remained constant, in contrast to Lorentz's (Janssen\&Mecklenburg, 2006, p. 80 - 81). The fourth account considered by Kaufmann was Einstein's, who in his (1905a, p. 919) article on the principle of relativity had also obtained equations for the electron's longitudinal and transverse mass that were equivalent to Lorentz's, as Kaufmann pointed out $(1906$, p. 491 - 492). In this way, the experiments promised not only a decision between different electron models, but also between different approaches to the foundations of physics: whereas Abraham's electromagnetic electron represented the electromagnetic world view, Einstein's and Lorentz's represented an approach that attempted to base the whole of physics on the principle of relativity (Kaufmann, 1906, p. 493). ${ }^{13}$

\footnotetext{
${ }^{13}$ Lorentz's work on the electron was seen as relativistic for two reasons: first, because it required non-electromagnetic forces to ensure its stability, it violated the electromagnetic program's aim to reduce the whole of physics to the laws of electrodynamics; and second, because his primary aim was to account for the null result of the Michelson-Morley experiment, i.e. the impossibility to detect any motion of the earth with respect to the ether (Janssen, 2009, p. 34). Bucherer's electron seems to escape this classification, but given that it didn't really play a role of importance in the Kaufmann episode, it will not be
} 
Relativistic treatment of the Kaufmann experiments. What sets Einstein apart from the rest, however, is that he does not rely on any specific electron model. For the time being, he rather takes the electron to be just a material point particle endowed with a certain electric charge. As such, Einstein leaves open the question of the electron's actual physical constitution (Einstein, 1905a, p. 917). Einstein rather proceeds on the basis of the principle of relativity and the light principle (1905a, p. 895): ${ }^{14}$

(PoR) The laws governing the changes of state of any physical system do not depend on which one of two coordinate systems in uniform translational motion relative to each other these changes are referred to.

(LP) Each ray of light moves in the coordinate system "at rest" with the definite velocity $V$ independent of whether this ray of light is emitted by a body at rest or in motion.

These principles allow Einstein to obtain transformation equations for the times, locations and electric and magnetic fields that ensure that the laws of mechanics, electrodynamics and optics obey the principle of relativity. To obtain equations for the velocity-dependence of mass, Einstein then applies the relativistic coordinate transformations to the equations of motion for such an electron. By making use of Newton's force law, he then obtains expressions for the electron's longitudinal and transverse mass, which are later identified with Lorentz's equations (1) with $l=1$ because of symmetry-

\footnotetext{
discussed further.

${ }^{14}$ All quotes by Einstein are taken from Anna Beck and Peter Havas's English Translation Supplement of the second volume of the Einstein Papers (1989).
} 
considerations. ${ }^{1516}$ A second aspect that sets Einstein apart is that his derivation is not even particularly concerned with the electron as such: the obtained equations apply not just to the electron in motion, but to all material bodies in general, since for the purpose of the derivation he takes the electron to be merely a material point endowed with an arbitrarily small charge (1905a, p. $920)$.

That Einstein proceeds in this way now shows, according to Janssen, that he correctly grasped that the phenomenon is kinematical in the broad sense of

\footnotetext{
${ }^{15}$ In fact, in his original article Einstein arrived at equations that differed slightly from Lorentz's, because he did not use a relativistic force-law, expressed in terms of momentum and conservation of energy, but rather the Newtonian force law, expressed in terms of mass and acceleration. This mistake was first picked up and corrected by Kaufmann (1906, p. 530 - 531) and corrected by Max Planck (1906a). Einstein himself first employed this new force law in his (1907c) review article on the theory of relativity. This is a bit of an ironic oversight, since Einstein himself was quite aware of the importance of the force-law employed in this context: right after his presentation of the mass-equations he in fact states that, with another definition of force, one would have arrived at different results, which entails that one has to be careful here (1905a, p. 919).

${ }^{16} \mathrm{On}$ the basis of his two principles, Einstein arrives at transformation equations relating two reference frames in relative motion, $K$ and $k$, which moves with a velocity $v$ with respect to $K$. These expressions, however, contain an expression $\phi$, a function of the velocity $v$ that is at that point in the derivation unknown. To clarify the meaning of this unknown term, Einstein then introduces a third reference frame moving with velocity $-v$ with respect to the frame $k$. This shows him that $\phi(v) \phi(-v)=1$. Reflecting on the length of a rod, at rest in $k$ and thus in motion with respect to $K$, then shows him that the length of the rod in $K$ is $l / \phi(v)$. Reasons of symmetry, he then claims, show us that the length of the rod does not change when $v$ is replaced by $-v$, which leads him to $\phi(v)=\phi(-v)$, which entails that $\phi(v)=1$ (Einstein, 1905a, p. $900-902$ ).
} 
being independent of the dynamics, and that dynamical investigations were "misguided[:] [a]s Einstein (1905a, §10) first suggested, Eq. (1) for $l=1$ simply gives the generic velocity-dependence of mass in a new relativistic mechanics. These relations provide no insight whatsoever into the origin of the electron's mass" (2009, p. 33). This is what Janssen takes to be the radical novelty of Einstein's STR.

As Janssen also points out, however, Einstein's account was not generally seen in this way at the time. For when Kaufmann, for example, presented the results of his latest series of experiments, he equated Einstein's account with Lorentz's dynamical electron theory, because their predictions were formally equivalent (see footnote 15) and because they had the same concern, i.e. to ensure that their theories obey the principle of relativity (Janssen, 2009, p. 34). ${ }^{17}$ Moreover, the results of these experiments were devastating for the Lorentz-Einstein equation: it came out worst of the three models considered in all three different analyses of his measurements that were carried out by Kaufmann. ${ }^{18}$ This led Kaufmann to the conclusion that it was definitively

\footnotetext{
${ }^{17}$ Kaufmann was not the only one who equated Einstein's and Lorentz's theories. Einstein himself, for example, also spoke about the theory of Lorentz and Einstein (1906c, p. 586), and Planck (1906b, p. 756) spoke about the Einstein-Lorentz theory. See Staley (2008, p. 301 - 309) for a discussion of the different ways in which many other scientists at the time also grouped Einstein together with Lorentz.

${ }^{18}$ Miller (1981, p. 230 - 231) and Hon (1995) offer an extensive discussion of Kaufmann's data-procedure. As Hon points out, "Kaufmann determined several constants of the curve that had been recorded on the photographic plate; he compared these constants with the corresponding constants obtained from the various theories" (1995, p. 190). In short, Kaufmann evaluated the different theories with respect to how their predictions differed
} 
shown that the attempt to base the whole of physics on the principle of relativity had failed (1906, p. 534).

According to Janssen, later experiments carried out by Bucherer (1908) produced results in favor of the Einstein-Lorentz equation, and these together with other experiments led to a broad consensus, according to Janssen (2009, p. 35), that the data were in fact closer to the Lorentz-Einstein prediction than to Abraham's. The central lesson to be learnt here for Janssen, however, is that we should not put too much weight on these experimental attempts to probe the velocity-dependence of mass, since most scientists "all paid lip service to the importance of the experiments, especially when the data seemed to favor their own theories [...], but it is not clear how seriously they really took them. I suspect that theoretical considerations eventually led to the acceptance of the Lorentz-Einstein formula" (2009, p. 35). We should not pay too much attention to the experimental results, according to Janssen, because STR shows, as Einstein already saw in 1905, that the idea to acquire insight into the constitution of the electron by investigating the velocitydependence of mass is "misguided" (2009, p. 33) and an "illusion" (2009, p. 35), since the phenomenon arises for all kinds of physical systems, not just for electrons. And while Einstein's insight that the phenomenon is purely kinematical could in 1905 still be seen as merely a theoretical suggestion, Max von Laue's continuum mechanics (1911a; 1911b) ${ }^{19}$ formulation of the

from the measured (a) curvature formed by the electrons on the photographic plate, (b) the charge-to-mass ratio of the electron's constituting Becquerel rays, and (c) the precise deflection of the electrons by the electromagnetic field in the $y$-direction.

${ }^{19}$ See (Janssen\&Mecklenburg, 2006, p. 68 - 71; $99-105$ ) and (Janssen, 2009, p. $35-$ 
theory definitely established this claim(2009, p. 35). This becomes clear once we derive the equations (1) for $l=1$ in this framework:

This derivation of these relations shows how generic they are. They give the velocity-dependence of the mass of any closed system described by Lorentz-invariant laws. ${ }^{20}$ Other than that, they reveal nothing about the system. It is because the electron model of Lorentz and Poincaré is closed and Lorentz invariant that their contractile electron obeys these relations; it is because Abraham's model is not Lorentz invariant that his rigid electron does not. (Janssen, 2009, p. 36)

In this way, STR establishes that the velocity-dependence of mass is a purely kinematical phenomenon, both in the broad and in the narrow sense. That the phenomenon is kinematical in the broad sense follows from the fact that the theory shows the equations to be generic: the phenomenon arises for any material system in motion and is thus independent of the specifics of the dynamics of the system displaying the behaviour, as Einstein, according to Janssen, already saw in (1905a), as we have seen on page $14 .^{21}$

37) for an extensive discussion of Laue's formulation of special relativity.

${ }^{20}$ For Janssen's definition of a closed system, see (2009, p. 35).

${ }^{21}$ It is important to stress here that this does not mean that Janssen claims that special relativity says all there is to say about mass tout court. As Janssen points out, if one wants to account for the origin of the rest mass $m^{\prime}$ of a particular system, one will of course have to rely on detailed dynamical considerations. Special relativity shows, however, that the velocity-dependence of mass of such a system in motion arises independently of such considerations, and as such the theory shows, according to Janssen, that the phenomenon is kinematical in the broad sense (Janssen, 2009, p. 38). 
The theory also shows that the phenomenon is kinematical in the narrow sense of exemplifying standard spatio-temporal behavior, according to Janssen. It does this by means of its Minkowskian space-time geometry, in which the Lorentz transformations which follow from the theory's postulates find what Janssen describes as their "natural interpretation" (2009, p. 39). What he means by this is that the theory tells us that whatever the fundamental laws of physics will be, their space-time geometry will be Minkowskian, or equivalently, the laws will be invariant under the Lorentz transformations. A phenomenon is then considered to be kinematical in the narrow sense if the system's behavior is a straightforward consequence of the Lorentz invariance of the laws that govern this behavior. We then say that the system exemplifies standard spatio-temporal behavior: the system's behavior reflects the geometry of Minkowski space-time because it is a consequence of the Lorentz invariance of whatever physical laws that actually govern this behavior. That the velocity-dependence of the electron's mass exemplifies such standard spatio-temporal behavior shows itself, according to Janssen, in the way in which Laue derives the equations 1 with $l=1$ (2009, p. 40$).^{22}$

\footnotetext{
${ }^{22}$ Janssen himself points out that Laue relies not only on the transformation equations for length and time, but also on the one for force, and that as such, one could claim that more is involved in the relativistic account of the phenomenon than purely kinematical considerations. According to Janssen, however, this is not correct, since the theory's central concepts reflect the Minkowskian space-time structure: "[r]elativistic continuum mechanics is tailored to the structure of Minkowski space-time. The behavior of its general concepts [...], under Lorentz transformations reflects this space-time structure. So even though the transformation law for mass cannot be established without reference to
} 
In this way, Janssen takes the Kaufmann episode to provide historical evidence for his more general claim that special relativity reveals the kinematical nature of the relativistic behavior of systems in motion: it is because the motion of these systems takes place in a space-time characterized in terms of Minkowskian geometry that they display this behavior, and that we need to describe this behavior in terms of Lorentz-invariant laws. In this way, he concludes, it becomes "clear that the relevant features of the moving system do not call for a dynamical explanation in special relativity[:] [t]hey are kinematical in the sense that the moving system is just exhibiting default spatio-temporal behavior" (Janssen, 2009, p. 48).

\section{The Principle of Relativity and the Kaufmann Experiments}

In what follows I will discuss the way in which some of the proponents of the principle of relativity responded to the Kaufmann experiments and the issues related to it. My aim with this is to argue against the historical narrative underlying Janssen's general claim that the theory of special relativity, already in Einstein's (1905a) formulation, established that the velocitydependence of mass is a kinematical phenomenon and that the relativistic study of this phenomenon is completely independent of the dynamics of the systems displaying such behaviour. I will show, more specifically, that such a conceptualization of the phenomenon is a consequence not so much of Einstein's (or Planck's) formulation of STR, but rather of Hermann Minkowski's re-interpretation of it. As such, the central consequence of my argument here

concepts over and above spatio-temporal ones, the velocity-dependence of mass is still kinematical, even in the narrow sense" (2009, p. 40). 
will be that we need to draw a significant distinction between what I will call Einstein's heuristic approach to the theory of relativity, and Minkowski's normative approach. ${ }^{23}$

In short, this distinction comes down to the following. On the heuristic approach, the principles of the theory of relativity primarily function as instruments that can help in the construction of relativistic theories concerning specific phenomena: this is done by applying the relativistic timeand length-transformations, which incorporate the two principles, to the laws that govern these specific phenomena. In themselves, however, these principles only suggest, but do not provide these theories yet: for this, a study of the dynamics of the systems underlying these phenomena is also required. On this heuristic approach, the range of the validity of the theory's principles is still an open question, and this can be tested experimentally. As a consequence of this, the principles can only be employed in a piecemeal way, by applying them to specific cases, since the principles do not provide a relativistic world view.

On the normative approach, on the other hand, the principles of the theory of relativity primarily function as norms for all existing and future

\footnotetext{
${ }^{23}$ The central aim of this historical discussion is thus to trace how the meaning of the principles of the theory of relativity, and the way in which they were used, changed over time in the early development of the theory. I will therefore refrain from giving a general definition of what I take to be these principles, since it is the differences between the different formulations that I am interested in. When discussing the views of particular authors, I have attempted, however, to clarify whether they are talking about the principle of relativity, the principles of the theory of relativity, or the theory and its consequences. I would like to thank an anonymous reviewer for pressing me on this issue.
} 
theories: they enable scientists to evaluate whether a theory adheres to the principles by checking whether the theory's laws guarantee invariance of the space-time interval with respect to the Lorentz group, and if not, it tells them how to correct the theory. On this approach, the theory's principles have exceptionless validity, and because of their formulation they are prior to any physical experience: they do not allow for a direct way to test experimentally whether the world we live in abides by these principles. ${ }^{24}$

To argue for this, I will focus on three central points of Janssen's historical argument for his general philosophical claim: (i) his assertion that Einstein already in 1905 saw the phenomenon's kinematical nature (Janssen, 2009, p. 33); (ii) his claim that it was mainly theoretical considerations that led to the acceptance of the Lorentz-Einstein equation (2009, p. 35); and (iii) his characterization of Minkowski's geometry as offering a natural interpretation of the theory $(2009$, p. 39). In this way, it will become clear that the precise interpretation of STR still needed to be elaborated after Einstein's (1905a) article, and that it is therefore difficult, from a historical-philosophical point of view, to speak about the theory establishing the kinematical nature of certain phenomena, as Janssen does. ${ }^{25}$

\footnotetext{
${ }^{24}$ As these descriptions indicate, the heuristic and the normative approach are not complete opposites: for both, the theory's principles can provide indications for future research. The main difference, however, is in what the adherents of the approaches prioritize in their use of the principles, and in their epistemological characterization of the principles: on the heuristic approach, they are open-ended, experimentally testable and the range of their validity is an open question; on the normative approach, on the other hand, they are universal, they have exceptionless validity, and they are prior to physical experience.

${ }^{25}$ Of course, this historical claim in itself is perfectly well be compatible with Janssen's
} 


\subsection{Einstein and Ehrenfest}

Ehrenfest and the deformable electron. My starting point for this will be an issue raised by Paul Ehrenfest (1907) concerning the relativistic treatment of the electron to which Einstein replied in an equally short note (1907a). The question raised by Ehrenfest concerns the same issue as Abraham had raised earlier for Lorentz's theory: the stability of the electron (see page 11). Abraham had shown, more specifically, that for a rigid ellipsoidal electron forcefree ${ }^{26}$ inertial motion was not possible in all directions, but only in the direction of its major axis. This entailed that it was unclear how force-free inertial motion was possible for a deformable charge distribution. Given that Einstein's theory forms what Ehrenfest calls a "closed system" 27 that in essence

more general philosophical claim about how the special theory of relativity should be interpreted: even though dynamical studies played a role in the historical development of the theory, the theory should in fact be interpreted as being concerned with the kinematical nature of the relativistic phenomena. I would have no problem with this response to the historical work presented here. Given, however, that Janssen explicitly states that his approach to issues in the philosophy of physics is based on historical considerations (see page 7 ), we can and should inquire into what the implications of the historical details could be for the more general philosophical issues. I would like to thank an anonymous reviewer for pressing me on this issue.

${ }^{26}$ Force-free here means, as we have seen on page 11, that the inertial motion could be maintained "without any external forces balancing the electron's self-forces" (Miller, 1981, p. 57$)$.

${ }^{27}$ Ehrenfest does not state explicitly what he means by this claim. He does specify, however, that this means that the theory should be able to provide, in a completely deductive way, an answer to his question. In general, Ehrenfest thus seems to see the theory of relativity as a theory that, with regards to a specific domain (here electrodynamics), should be able to account for all phenomena belonging to this domain. 
offers a reformulation of the "Lorentzsche Relativitäts-Elektrodynamik", ${ }^{28}$ it should be possible, according to Ehrenfest, to deduce an answer from the theory to Abraham's issue, formulated in the following terms: let us assume, with Einstein, that we have a deformable electron, to which we do not ascribe any particular form at rest $;^{29}$ the question then is: is force-free inertial motion possible in any direction for such a deformable electron? (Ehrenfest, 1907, p. $204-205)$.

The issue raised by Ehrenfest, as he himself points out, exactly concerns what is at stake in the Kaufmann experiments: the dynamics of the electron. Regarding this issue, Ehrenfest then points out that the theory of relativity as a closed system can provide two different answers in its current formulation, both of which are equally problematic. If it tells us that such motion is not possible in all directions for the deformable electron, then a new hypothesis should be added to the theory that explicitly excludes the existence of such electrons, for otherwise it would be possible to detect absolute rest. If it is

\footnotetext{
${ }^{28}$ Ehrenfest thus describes Einstein's theory of relativity as an electrodynamical theory, primarily a reformulation of Lorentz's electrodynamics. Ehrenfest refers here not to Einstein's original STR-article (1905a), but rather to the short paper (1905b) which contains Einstein's derivation of the energy-mass equivalence. We should not see Ehrenfest's description of the theory as an electrodynamical one as a case of bad interpretation of the theory: Einstein himself described his derivation of the energy-mass equivalence as a very interesting consequence of what he calls his (1905a) electrodynamical investigations (1905b, p. 639).

${ }^{29}$ Ehrenfest refers here to a statement made by Planck (1906a, p. 137), that his relativistic dynamics allows us to obtain a precise determination of the dependency of inertia ("Trägheit") on velocity without the need to specify the form of the electron.
} 
possible, then it should be shown how this follows from the theory without introducing a completely new axiom (Ehrenfest, 1907, p. 205). What this shows is that, at the time, Kaufmann's experiments were taken to pose the dynamics of the electron as an issue to which electrodynamical theories had to provide an answer. Moreover, this issue not only concerned theories of the electron such as e.g. Lorentz's, but equally well Einstein's theory of relativity. Even given its new, principled formulation, the electron was still taken to raise issues for the theory. As Staley puts it: "Ehrenfest's question shows him taking the epistemological demands of relativity seriously, holding the theory to its own standards" (2008, p. 264). ${ }^{30}$

Einstein's response to Ehrenfest. Einstein's (1907a; 1907b) response to Ehrenfest's note now shows that he took the dynamics of the electron to pose an actual issue to which the theory of relativity had to provide an answer. In his short direct reply to Ehrenfest, he sketches how the theory could provide a response to this issue. He starts by stating that the principle of relativity and the light postulate should not be taken as forming a closed system, or

\footnotetext{
${ }^{30}$ Staley himself does not specify which epistemological demands he has in mind. Given the way in which Ehrenfest formulates his question, we can infer, however, that he took the following elements to be significant epistemological characteristics of the way in which Einstein formulated his theory: that Ehrenfest asks how the theory can answer his question without introducing new axioms shows that he recognized the importance of principles in Einstein's formulation of the theory; Ehrenfest also saw that the theory's most important point was the exclusion of the detection of absolute rest; and that he specifies that the issue arises independently of the electron's precise form shows that he saw what was peculiar about Einstein's relativistic approach to the electron, which did not involve a specific electron model.
} 
even as a system at all. They should rather be seen as offering merely a heuristic instrument, which in itself only makes claims about the physical behaviour of rigid bodies, clocks and light signals, i.e. the physical entities and processes employed to carry out measurements of lengths and times in inertial frames of reference. ${ }^{31}$ The theory only leads to statements about other physical systems if these principles are applied to the laws of physics, in which case they can lead us to previously unknown relations between these laws. The theory's principles in themselves, however, do not lead us to what Einstein calls "additional statements" besides those concerning rods, clocks and light signals (Einstein, 1907a, p. 206).

Einstein then sketches how such a heuristic use of the principles constituting the theory works, by showing how it allows one to obtain a relativistic theory of the electron's motion: by applying the relativistic time- and lengthtransformations to the Maxwell equations for empty space, one can obtain transformation equations for electric and magnetic forces; employing these, together with the time- and length-transformations, then leads one to the laws of motion for the electron. What is important here, is that the principles, in their current formulation, should not be seen as forming any kind of closed system which already contains, in a deductive way, answers to questions concerning the electron itself: the principles only provide instruments

\footnotetext{
${ }^{31}$ Janssen dismisses this reading of the theory, because he takes it to be an idiosyncratic reading stemming from Einstein's operationalist preferences at the time; the theory is in fact, according to Janssen (2009, p. 28), really concerned with the default spatiotemporal behavior of all physical systems, which, he claims, becomes especially clear in the Minkowskian formulation of special relativity.
} 
for the formulation of theories that could address such questions.

Einstein does not stop here, however. In the second part, he provides a sketch of how one could obtain a deductive answer to Ehrenfest's issue regarding the constitution of the electron. He starts by pointing out that the electromagnetic program assumes that the electron forms a rigid structure over which electricity is distributed, but that the laws governing the motion of such a structure are not deducible from electrodynamics on its own. This entails that a deductive answer to the issue of the electron's dynamics can only be formulated when one possesses a sufficiently accurate dynamics of the rigid body. However, from a relativistic point of view, Einstein then points out, such a theory has not been obtained yet:

If the theory of relativity is correct, we are still far from the latter goal [i.e. a dynamics of the rigid body]. For the time being, we only have the kinematics of parallel translation and an expression for the kinetic energy of a body in parallel translation, provided the latter does not interact with other bodies; for the rest, both the dynamics and the kinematics of a rigid body have at present to be considered as unknown. (Einstein, 1907a, p. $207-208$ )

What this indicates is that, according to Einstein, neither an electromagnetic approach, nor a relativistic one, could address Ehrenfest's issue in a completely satisfying way at the time, since neither could provide, on its own, a sufficiently accurate dynamics of the rigid body. What is equally important here, however, is that Einstein states that the theory of relativity could not answer this question as of yet ("noch weit entfernt"), which suggests that he saw Ehrenfest's issue as one that needed to be addressed. If 
that is the case, we have an indication that, contra Janssen's claim, Einstein did not consider his (1905a) treatment of the velocity-dependence of mass to be the final, kinematical word on the issue. In what follows, I will argue that this is indeed the case, and that this suggested to Einstein that addressing this issue required a relativistic study of the dynamics underlying systems displaying the phenomenon.

Einstein and Relativistic Dynamics. Evidence for this can be found in another article by Einstein from this period (1907b), which concerns the inertia of energy. ${ }^{32}$ Einstein's starting point here is the result obtained in his (1905b) article to which Ehrenfest responds, i.e. that the principles of the theory relativity in combination with the Maxwell equations entail that the inertia of a body changes in a determinate way with its energy content (Einstein, 1907c, p. 371).

Einstein immediately points out, however, that up until now this general claim has been elaborated solely on the basis of considerations pertaining to very specific cases. ${ }^{33}$ This necessitates a more general investigation of the precise scope and justification of this energy-mass dependency. As of now, however, a general answer to this question cannot be given, because we do not yet have a world view in conformity with the principle of relativity ("ein vollständiges, dem Relativitätsprinzip entsprechendes Weltbild") (1907c, p. $371-372)$. Any investigation of the energy-mass dependency is therefore for now obliged to proceed by means of specific cases in the domain of relativistic

\footnotetext{
${ }^{32}$ See Miller (1981, p. 236 - 238) for a more extensive discussion of this paper.

${ }^{33} \mathrm{He}$ refers here to his (1906a) article on the principle of the constancy of centre-of-mass motion.
} 
electrodynamics, since in that domain we are on secure ground. At the same time, however, Einstein is also well aware of the limitations of proceeding in this way, since he points out that his recent research on radiation, specific heats, and the light quantum has shown that the Maxwell equations have only limited validity. ${ }^{34}$ In the case considered, however, this does not pose a problem, since it does not concern these specific phenomena.

What is interesting about these remarks is that they elaborate how Einstein saw the heuristic approach to the theory of relativity. Applying the principles of the theory to the electrodynamic laws governing specific cases leads to what he called, in the reply to Ehrenfest, "additional statements" (Einstein, 1907a, p. 206), such as the statement that there is a specific massenergy dependency. Given, however, that at the time no relativistic world view is available yet, the only way to investigate further such claims is by a piecemeal approach, i.e. by applying the principles to specific issues in electrodynamics, since it is only there that the principles find a secure ground. Only once the theory also covers quantum phenomena will we be in a position to account, in a general way, for the additional statements obtained by applying the principles to specific cases.

After these remarks on a future relativistic world view, Einstein then starts, in $\S 1$, with a discussion of the way in which the kinetic energy of a rigid body in uniform translation subject to external forces is handled by relativity theory $(1907 \mathrm{c}$, p. $373-377)$. Applying these insights, in $\S 2$, to electrically charged rigid bodies then brings Einstein to an equation for

\footnotetext{
${ }^{34}$ Einstein refers here to some of his earlier articles on light quanta: (1905c), (1906b), (1907d).
} 
their kinetic energy which differs, he points out, from the equation obtained earlier for the kinetic energy of a non-charged rigid body. This entails that an electrostatically charged body possesses an inertial mass that surpasses that of a non-charged body, and as such this special case provides evidence for the inertia of energy (1907c, p. 379).

In $\S 3$, Einstein then turns his attention towards a relativistic treatment of the dynamics of the rigid body, the attainment of which, he claims, could seem to be near at hand on the basis of $\S 1$ and $\S 2$. He warns, however, that the claims of $\S 1$, on which the results of $\S 2$ are based, only hold for situations where the forces involved are constant over time. If this is not the case, he shows by means of a simple example, we are led to results that would contradict the work-energy principle. After showing that this problem cannot be resolved, in relativistic terms, by appealing to the instantaneous spread of force, he is then brought to restate his answer to Ehrenfest's question (see page 27): "if relativistic electrodynamics is correct, we are still far from having a dynamics of the parallel translation of the rigid body" (1907c, p. 381). In the final section, Einstein then concludes with a discussion of the energy of a system of mass-points in force-free motion, a special case that, again, provides evidence for the general claim about the inertia-energy relation stated at the beginning of the paper (1907c, p. 384).

What is of interest here now are not primarily the results obtained by Einstein, but rather the choice of topics: the kinematics and dynamics of the (charged) rigid body. This is surpisingly in line with Einstein's response to Ehrenfest's challenge that, at that time, both a kinematics and dynamics of the rigid body for the electron were to be considered as unknown (see page 
27). As such, this indicates that Einstein was actually concerned with the issue raised by Ehrenfest concerning the dynamics of the electron, and that he attempted to find a suitable relativistic answer to it. Moreover, the paper offers a good illustration of how Einstein employed the principle of relativity as a heuristic instrument for investigating such issues: it is through the application of the principle of relativity and the light postulate, incorporated in the relativistic time- and length-transformations, to particular laws of physics - in this case the principle of the conservation of energy and the Maxwell equations, with the scope restriction discussed above - that we can obtain further statements about a particular domain - in this case statements about the inertia of energy in the domain of relativistic electrodynamics.

At the same time, the paper also shows that the application of the principles of the theory of relativity to the laws of physics should not be seen as the final word: as Einstein points out in the introduction, a final account will have to be formulated in terms of a view that can accommodate the insights obtained in his work on quanta and light- and radiation-phenomena. As such, it is clear that Einstein was well aware that there were limitations to the way in which relativity theory was formulated at the time, but also that he believed that these limitations could be overcome: while he now still needed to proceed by means of particular cases, he does seem to indicate in the introduction that the attainment of a complete world view corresponding to the principle of relativity is possible.

This now shows that Einstein considered the issue raised by Ehrenfest, concerning the relativistic view on the dynamics of the electron, to be pressing and important. As such, this provides an argument against Janssen's claim 
(i) that Einstein already in 1905 saw that the velocity-dependence of mass was a purely kinematical phenomenon, and that a relativistic study of it is independent from the dynamics of the system displaying the behaviour. It also shows that, in its present form of a piecemeal heuristics, the principle of relativity and the light postulate in themselves were not suited to provide a complete answer to the question. In order to understand how a relativistic reply to this issue raised by Kaufmann's experiments and the electromagnetic program eventually did come about, ${ }^{35}$ we will now turn to Planck's work on the experiments and his general dynamics.

\subsection{Relativity, Radiation and the Electron}

We turn now to Planck because it is mainly his work that allowed for the elaboration of a relativistic response to Kaufmann's experiments and the electromagnetic program. We will focus here, more specifically, on four of his articles in which he discusses these issues explicitly: his (1906a) elaboration of a relativistic mechanics; his (1906b) analysis of Kaufmann's experiments; his (1908a) elaboration of a general dynamics; and his (1908b) discussion of the principle of action and reaction. ${ }^{36}$ In this way, it will also become clear how

\footnotetext{
${ }^{35}$ In his (1907c), Einstein does not refer to the results of the Kaufmann experiments, nor does he mention any of the existing conceptions of the electron. He does refer to Kaufmann once, but not with regards to his experiments. Einstein rather remarks that he will probably repeat claims made earlier by other authors, but that he should be pardoned for not offering an overview of the existing literature. He then expresses the hope that others will fill up this gap, as has been done by Kaufmann and Planck for his (1905a) relativity article (1907c, p. 373).

${ }^{36}$ Planck's direct engagement with these issues stands in stark contrast with Einstein's, who only explicitly refers to Kaufmann's experiments in his (1907b) review article. Ein-
} 
Planck's work provided the context in which a tentative relativistic response to Ehrenfest's challenge could be elaborated by Einstein (1909a; 1909b).

Planck's relativistic mechanics. Planck first discusses Kaufmann's results in his (1906a) elaboration of a mechanics on the basis of the principle of relativity, which he presents as the claim that

none of two frames of reference $(x, y, z, t)$ and $\left(x^{\prime}, y^{\prime}, z^{\prime}, t^{\prime}\right)$ related by the relativistic transformations for lengths and times can be considered as the rightful frame of reference for the fundamental equations of mechanics and electrodynamics, and none of them can thus be called "at rest". (Planck, 1906a, p. 136; personal translation)

Such an elaboration is required, according to Planck, because the application of the principle of relativity to Newtonian mechanics, in its formulation in terms of mass and acceleration, renders the relation between kinetic and potential energy very complex (1906a, p. 137 -138). He therefore proposes, on the basis of a relativistic treatment of the force exerted by an electromagnetic field on a mass point endowed with a charge, to reformulate the equations of mechanics in terms of the rate of change of momentum. ${ }^{37}$ This

stein does refer implicitly, however: in his (1905a, p. 919), he derives a prediction for the electron's longitudinal and transverse mass, and in his (1906c) he proposes an alternative kind of experimental set up, involving cathode rays, to determine the velocity-dependence of the electron's mass.

${ }^{37}$ As an interesting aside, this discussion clearly shows how Planck, as Einstein did, also employed the principle of relativity as a heuristic principle: it is the application of the principle of relativity and the light postulate, in terms of the relativistic time- and lengthtransformations, to the equations for the charged mass point's electric and magnetic fields 
reformulation, as he shows in his concluding remarks, can also be captured in Lagrangian and Hamiltonian terms (1906a, p. 139 - 141). ${ }^{38}$

This reformulation, as we will see later, will play an important role in Planck's analysis of Kaufmann's experiments. It is not, however, the only important aspect of this article. Equally significant is Planck's introduction, in which he discusses the issues raised by Kaufmann and the electromagnetic program. He starts by pointing out that the principle of relativity, introduced by Lorentz and generalized by Einstein, has led to a great simplification of the electrodynamics of moving bodies. At the same time, the question of its acceptability as a general, all-encompassing principle for physics also seems to have been answered recently, by Kaufmann, in negative terms. We should not, however, judge too rapidly, for the theory will eventually, according to Planck, be brought in agreement with observation. Moreover, the least we can do for a physical account that promises such simplicity and generality is to investigate it as far as possible, even in the eye of fundamental challenges.

One of these challenges is the question of the work involved when a moving electron undergoes deformation according to the principle of relativity, which we can put aside for now, according to Planck, since it can be accounted for in terms of the electron's kinetic energy. One particular advantage of proceeding

that shows him how to reformulate the equations of motion. See Elie Zahar (1989, p. 227 - 237) for a thorough discussion of Planck's paper.

${ }^{38}$ Miller (1981, p. 327 - 331) discusses how it was this reformulation by Planck that corrected Einstein's use of Newtonian mechanics in his derivation of the equations for the electron's longitudinal and transverse mass, which showed that the relativistic equations were in fact identical to Lorentz's for $l=1$ (see footnote 15). 
in this way, he then points out, is that the issue of the electron's precise form is no longer of any real importance: it does not matter whether we ascribe it a spherical form or any determinate form at all in order to calculate the electron's velocity-dependence (1906a, p. 137). ${ }^{39}$ What this shows is that it is only by putting aside the issues that are of central importance for the electromagnetic program - Kaufmann's results and the constitution of the electron - that Planck is able to open up a space for the further investigation of the physical implications of the principle of relativity. In this way, it becomes clear how pressing the challenge posed by the Kaufmann experiments was for the theory of relativity at the time.

Planck and the Kaufmann experiments. It seems, however, that attempts to carve out a space for the elaboration of the theory of relativity would not really have been visible as long as the theory's adherents did not address what was taken to pose a direct challenge to the theory at the time, namely Kaufmann's experimental results. To overcome this, Planck (1906b) therefore undertook a critical assessment of Kaufmann's experimental set-up and data. ${ }^{40}$ After praising Kaufmann's precision and clarity, Planck starts by outlining the main difference between Kaufmann's original analysis and

\footnotetext{
${ }^{39}$ It is this claim that Ehrenfest picks up on later, as we have seen on page 24: according to Ehrenfest, even when we refrain from ascribing a particular form to the relativistic electron, its dynamics still poses an issue for the theory of relativity, since the theory is not clear on whether inertial force-free motion is possible for such an electron in all directions.

${ }^{40}$ Cushing (1981, p. 1142 - 1146) and Zahar (1989, p. 201 - 226) both offer a very extensive discussion of the work undertaken by Planck in this paper.
} 
his re-analysis: while Kaufmann reduced the measured deflections $(\bar{y}, \bar{z})$ to infinitely small deflections $\left(y^{\prime}, z^{\prime}\right)$ in order to simplify the calculations required (Staley, 2008, p. 240), Planck will not carry out such a reduction. ${ }^{41}$ He will work with fully integrated equations for the electron's motion, which requires him to express them as Lagrangian functions in terms of the kinetic potential $H$, in line with his (1906a) article. Combining these with the electromagnetic field values involved, Planck then provides his expressions for the electric and magnetic deflection in terms of the momentum $p=\partial H / \partial q$, where $q$ is the electron's velocity. Applying these expressions to the values obtained by Kaufmann then allows him to evaluate what he takes to be at stake in the experiments: the way in which the velocity-dependence of the electron's momentum brings about the measured deflections $\bar{y}$ and $\bar{z}$. On the basis of this it is then possible to compare the two theories involved, Abraham's 'Kugeltheorie'42 and the Lorentz-Einstein 'Relativtheorie', with respect to the different equations of motion they offer for the electron (1906b, p. 756).

On the basis of this reconceptualization of Kaufmann's experimental setup, Planck then draws evaluative comparisons on two different levels. The first concerns the two theories involved, and how their predictions fare with

\footnotetext{
${ }^{41}$ The application of magnetic and electric fields influences the electrons constituting the rays employed in the experimental set-up in such a way that they deviate from their normal path, which gives rise to the curved line on the photographic plate. See (Cushing, 1981, p. 1138), (Hon, 1995, p. 179 - 180) and (Staley, 2008, p. 225) for a more techical exposition of Kaufmann's experiments and his data-analysis.

${ }^{42}$ This name stems from the fact that Abraham conceived of the electron as a sphere ("Kugel").
} 
respect to Planck's re-interpretation of Kaufmann's results in terms of the velocity-dependence of the electron's momentum $p$. The second level concerns the calculation-method employed to analyse the data: Planck's use of fully integrated equations versus Kaufmann's use of infinitely small deflections. With regards to the second level of comparison, Planck immediately points out that there is no significant difference. And while, with regards to the first level, the Kugeltheorie is closer to the results than the Relativtheorie, we should not take this as definite evidence in favor of Abraham's theory. To make that claim, Planck argues, would require that the difference between Abraham's theory and the observed results would be small compared with the difference between relativity and observation, and that is not the case (1906b, p. 757). Planck then argues that the only reasonable conclusion to be drawn from Kaufmann's results at the moment is that more work needs to be done if we want these kinds of measurements to function as a definite arbiter between the two theories (1906b, p. 758).

Planck then sketches how this could be done, via a discussion of the assumption underlying Kaufmann's experiments that the biggest distinction between the two theories is to be found in the high-velocity spectrum. By means of his reformulation of the magnetic and electric deflection in terms of the momentum $p$, he argues, however, that this is not necessarily the case: in fact, it would be better to perform measurements on cathode rays, which have a lower velocity than the Becquerel-rays employed by Kaufmann (Planck, 1906b, p. 758). The advantage of employing cathode rays, according to Planck, is that they have a third measurable characteristic besides their magnetic and electric deflectability: their discharge potential $P$. He then 
sketches, as a conclusion to his paper, the way in which the different theories offer a prediction for this quantity in terms of the radiation energy $E$ (1906b, p. 759).

We are now in a position to see how Planck attempted to defuse, by different means, the direct challenge posed by Kaufmann's results. First, there is his re-formulation of the issues at stake in the experiments. No longer are they necessarily concerned with determining the electron's longitudinal and transverse mass in order to obtain insight into their rigid or deformable constitution; we can equally well read them as being concerned with determining the electron's momentum, in order to obtain insight into the electron's equations of motion. This is not just an innocent reformulation, for if we read it in combination with Planck's (1906a) paper, it becomes clear that underlying this reformulation there is in fact a certain preference for a mechanics more in line with the principle of relativity. ${ }^{43}$

Second, we have Planck's proposal of an alternative to Kaufmann's reliance on infinitely small deflections. The central aspect of this proposal is that it allows Planck to introduce his reformulation of the experiments in terms of the kinetic potential $H$ and the electron's momentum $p$, which, he shows, leads to an analysis of Kaufmann's data that performs equally well

\footnotetext{
${ }^{43}$ I claim that this reformulation is not innocent because, as Planck himself states in his (1906a, p. 137) article, his foremost reason for reformulating the equations of mechanics was not because of any particular problem, but rather because it would make the employment of the principle of relativity less complex. This is not an innocent move because this was, of course, exactly what was at stake in the debates over the Kaufmann experiments, i.e. whether it was possible to apply the principle in such cases.
} 
as Kaufmann's. In this way, Planck opens up the possibility of disputing Kaufmann's interpretation of the data, since he can now argue that there is an alternative interpretation which does not lead to the conclusion that the data argue for Abraham's theory, but rather to the conclusion that more work needs to be done.

Finally, we have Planck's suggestion of employing cathode rays. Kaufmann worked with high-velocity Becquerel-rays, because the velocities attainable by means of cathode rays were too small to showcase any noticeable mass increase, and as such these rays were not useful for investigating what he was interested in, i.e. the velocity-dependence of mass (1906, p. 488). Planck's interest, however, is not primarily with the electron's mass: as we have seen, he proposed to reformulate the theoretical issues at stake in the experiments in terms of the electron's momentum and kinetic potential. As such, the proposal to perform experiments on cathode rays forms part of Planck's attempt to shift the focus of the debate, while it also allowed him to present the principle of relativity as open to experimental testing. ${ }^{44}$ In this way, we see how Planck attempted, through his analysis of the Kaufmann experiments, to defuse the challenge posed by Kaufmann's results by shifting the focus of the debate away from those issues that were of central concern to the adherents of the electromagnetic program.

\footnotetext{
${ }^{44}$ Planck was not the first to present cathode ray experiments as an alternative to Kaufmann's experiments: Einstein (1906c) had already done the same, in a much more detailed way, and he repeats this proposal in his (1907c) review article. The main difference, however, is that Einstein still presented these experiments as primarily concerned with the electron's longitudinal and transverse mass.
} 
Planck's General Dynamics. Einstein (1907a; 1907b) had to admit that the principles of the theory of relativity could not yet offer a completely satisfying answer to Ehrenfest's question concerning a relativistic dynamics of the electron. Planck (1908a; 1908b) is the first to improve on this, in his work on what he calls a general dynamics: a dynamics that, constrained by the principle of relativity, covers not only mechanics in the narrow sense, ${ }^{45}$ but also thermodynamics and electrodynamics (1908b, p. 728).

What is new here, however, is that Planck does not proceed by means of electrodynamic cases, as was customary in earlier elaborations of relativity. He rather turns to thermal radiation phenomena, which concern the behavior of physical systems that are devoid of matter and only contain electromagnetic energy, but which nonetheless obey the fundamental laws of mechanics and thermodynamics (Planck, 1908a, p. 1). Planck's main reason for considering such systems is that they raise particular problems for some fundamental assumptions taken for granted up until now in the field of dynamics, of which he offers three examples.

The first is the belief that the total energy of a moving ponderable body consists of the sum of its kinetic energy (which depends only on the body's velocity) and its internal energy (which depends on the bodies density, temperature, and chemical composition). Recent investigations have shown, however, that such bodies also contain a finite amount of thermal radiation, for which it is impossible to distinguish these two kinds of energy if the body is in

\footnotetext{
${ }^{45}$ With this Planck means Newtonian mechanics, which he also often describes as 'pure' or 'normal' mechanics in the article. We will return to how Planck saw the relation between the theory of relativity and normal mechanics on in footnote 51 .
} 
motion. The distinction between kinetic and internal energy is therefore not tenable anymore across the board (Planck, 1908a, p. $1-3$ ).

The second is the assumption that a body's inertial mass ("träge Masse") forms an absolute, fundamental, and unchangeable element of physics, which is most directly defined, Planck points out, in terms of the body's kinetic energy. In the case of thermal radiation, however, it becomes clear that this constancy assumption cannot be maintained: the energy of such radiation, which depends on the system's temperature, contributes a part - which, following the previous assumption, cannot be grouped under either kinetic or internal energy - to the system's inertial mass, which entails that this mass becomes temperature-dependent (1908a, p. 3). ${ }^{46}$

The final concerns the assumed general identity between inertial and ponderable mass. ${ }^{47}$ The issue is that, while thermal radiation phenomena clearly have inertial mass, it is difficult to ascribe them ponderable mass. This issue in particular shows that a new general dynamics is called for, since it shows the possibility of rigid bodies whose laws of motion completely differ from

\footnotetext{
${ }^{46} \mathrm{An}$ important consequence of this for our story here, which will be discussed later on, is that this also diminishes the physical significance of the distinction between 'true' and 'apparent' mass (1908a, p. $3-4$ ). See footnote 11 for a short discussion of this distinction.

${ }^{47}$ Planck does not specify what he means by ponderable mass. Given his reconceptualization of mass in terms of energy at the end of the article, however, it seems that what Planck is trying to argue here is that a distinction needs to be made between the ponderable mass of material bodies and inertial mass, since radiation energy does have mass but is not ponderable in the sense of material. For more discussion of the different concepts of mass that were around during this period, see chapters 11, 12 and 13 of Max Jammer's (1961, p. $136-190)$.
} 
those of ordinary mechanics (Planck, 1908a, p. 4).

A thorough revision of the foundations of dynamics is therefore required, which, according to Planck, should be guided only by those principles that can claim exact validity in the face of these new findings. The first principle employed will be the principle of least action, which, as Hermann von Helmholtz had shown in (1895), comprises mechanics, electrodynamics and thermodynamics in their application to reversible processes. ${ }^{48}$ This is not yet sufficient, however, since the principle in itself does not yet lead us to a replacement for the defective distinction between kinetic and internal energy. This can be achieved, however, by making use of the principle of relativity, which Planck expresses in more or less the same terms as in his (1906a) (see page 3.2): the relativistic time- and length-transformations entail that there is no more need for one specific rest-frame for the laws of mechanics, electrodynamics, and, he adds now, thermodynamics. He now also adds the light postulate by stating that $c=c^{\prime}(1908 \mathrm{a}, \mathrm{p} .12)$.

Planck starts his study of a general dynamics constrained by these two principles by turning to a particular phenomenon, black-body radiation. ${ }^{49}$

\footnotetext{
${ }^{48}$ Reversible processes, as Planck himself points out a bit later in the article, are those changes to a system that are so slow that, for each instant of time, the system is in a stationary state (1908a, p. 7). What Helmholtz had shown, according to Planck, is that when the principle of least action is applied to changes of a body's state that are reversible, one can obtain differential equations for a body's pressure $p$, entropy $S$ and the force exerted on it expressed in terms of the body's kinetic potential $H$, its velocity $q$, its volume $V$ and its temperature $T$ (Planck, 1908a, p. 10). As we will see, this characterization of a body's state will prove central to Planck's formulation of his relativistic dynamics.

${ }^{49} \mathrm{~A}$ black body is a "cavity with perfectly absorbing (i.e. black) walls", which, if its
} 
His reason for this is that it is the only physical system for which we can specify its thermodynamical, electrodynamical and mechanical properties with absolute precision, independently of any conflicts between these theories (Planck, 1908a, p. 7). On the assumption that all changes occuring to the system are reversible, its state can be completely characterized in terms of its velocity $q$, its volume $V$ and its temperature $T$. Planck then shows how to express the system's momentum $G$, its energy $E$, and its pressure $p$ in terms of these independent variables, and how this leads to the first and second law of thermodynamics for black-body radiation (1908a, p. 7 - 9).

On the basis of this particular case, Planck then turns to the dynamics of material bodies in general. ${ }^{50}$ The state of such a system can equally well be determined in terms of the independent variables $V, T$, and the velocitycomponents $\dot{x}, \dot{y}, \dot{z}$. When the state of such a system is changed in a reversible way (see footnote 48), the principle of least action then provides us with expressions, in terms of the system's kinetic potential $H$, for the force it experiences, its pressure and its entropy.

Planck then points out that, up until now, it was assumed that the sys-

temperature is kept steady at specific temperature, will be filled with radiation energy: this is black-body radiation (Kuhn, 1978, p. 3). At the time, the physical study of this phenomenon was concerned, more in particular, with investigating how the intensity of such radiation depended on wavelength or frequency (Gearheart, 2002, p. 170). It was also in this domain that the concept of the quantum was first developed by Planck. For some extensive historical work on this episode, see the references in footnote 54 .

${ }^{50} \mathrm{He}$ conceives of such bodies as consisting of a definite number of molecules, which can be either identical in kind or different, and possibly zero in number, in which case we obtain the black-body case. 
tem's kinetic potential consisted of two parts, its internal energy $F$ (independent of $q$ ), and its constant mass $M: H=\frac{1}{2} M q^{2}-F$. If we hold on to this assumption, the principle of least action will lead us to the equations of what he describes as ordinary mechanics and ordinary thermodynamics (Planck, 1908a, p. 11) ${ }^{51}$. Thermal radiation phenomena have shown, however, that this distinction between two kinds of energy is no longer tenable (see page 40). To overcome this, Planck then turns to the principle of relativity. He employs this principle, together with the principle of least action, to investigate how the physical quantities discussed above (entropy, temperature, volume, pressure, ... ) transform between different frames of reference (1908a, p. 13 - 17). This then leads Planck to the transformation equations for the kinetic potential $H$ (1908a, p. 17).

It is this last relation that is of central importance for Planck, since in combination with the principle of least action, it allows us to determine the way in which the values of any physical quantity are related between two reference frames in relative motion (1908a, p. 20). This result not only leads him to a whole list of invariant properties (1908a, p. 23), but also to the following claim: the kinetic potential $H$ and all state variables of the systems under consideration here can all be specified as functions of their velocity, volume and temperature, under the condition that they are

\footnotetext{
${ }^{51}$ Here we thus see how, for Planck, normal mechanics is to be seen as an approximation of his relativistic dynamics: for those cases where the internal thermal radiation of a body makes no significant difference, we can assume the distinction between kinetic and internal energy, in which case the principle of least action will lead us to the laws of normal mechanics (Planck, 1908a, p. 11).
} 
known as functions of the system's volume and temperature for velocity zero (Planck, 1908a, p. 24).

Planck then turns to some of the implications of these results. Of particular importance here is his discussion of the concept of inertial mass. This quantity is determined by a body's momentum, which entailed, in pure mechanics (see footnote 51), that a body's inertial mass was seen as a constant of fundamental importance. According to Planck's general dynamics, however, it is a notion of only secondary importance: since momentum is no longer directly proportional to velocity, a body's mass no longer constitutes a constant. One particular consequence of this, Planck points out, is that the velocity-dependence of mass becomes primarily an issue of definition ("Definitionssache"): depending on how one relates mass to momentum, one obtains a different velocity-dependence. ${ }^{52}$

Planck then shows that, starting with a particular definition, which takes mass as the ratio of momentum and velocity $(M=G / q)$, one can obtain the expressions for a system's longitudinal and transverse mass. He immediately points out, however, that this definition cannot be the final word, since it cannot be tested directly, for example, by thermodynamic means. ${ }^{53}$ In fact,

\footnotetext{
${ }^{52}$ Planck lists a few possibilities: one option conceives of mass as the ratio of the momentum to the velocity $G / q$; another option is to differentiate the velocity $q$, in which case there are further options depending on how one differentiates; still another possibility is to represent a body's mass not in terms of momentum but in terms of its energy $E$ differentiated to $q^{2} / 2$ (1908a, p. 27).

${ }^{53}$ This elaboration also makes clear that these notions of longitudinal and transverse mass should no longer be seen as fundamental in any sense, as they were when Abraham first conceptualized them (see footnote 11). Rather, depending on how one differentiates
} 
further analysis of this definition within the framework of his general dynamics shows that it is easier to leave aside questions of how to define a body's mass, and that we should rather conceive of physical bodies as consisting fundamentally of energy (1908a, p. $27-29)$.

In this way, it becomes clear how particular dynamical considerations concerning the black-body played an important role in the elaboration of Planck's general dynamics. The reason why black-body radiation is so suitable for these kinds of investigations is exactly the fact that the phenomenon embodies that which Planck (and Einstein) considered to be central to a relativistic approach to physics, i.e. that it brings together all kinds of physical laws (mechanics, thermodynamics, electromagnetism, ... ) under a few general principles. It is because the phenomenon's constitution involves these different physical laws that its dynamics can inform us about how a general dynamics should look like from a relativistic point of view.

It also shows clearly how the elaboration of a relativistic response to the issues raised by Kaufmann's experiments equally well relied on dynamical considerations: it is through the study of the inertial mass of black-body radiation that Planck is led, in the end, to claim that the velocity-dependence of mass is primarily an issue of definition. The real difference with the electromagnetic approach is therefore not, as Janssen claims, that the relativistic

this ratio, one can obtain, in the case of black-body radiation, at least four different notions of velocity-dependent mass: the moving system's transverse mass; its longitudinal isothermal-isochoric (constant temperature and constant volume) mass; its longitudinal adiabatic-isochoric (no heat transfer and constant volume) mass; and its longitudinal adiabatic-isobaric (no heat transfer and constant pressure) mass (Planck, 1908a, p. 28) 
study of the velocity-dependence of mass was independent of any dynamical considerations. The difference rather concerns the kind of dynamical system considered: whereas Abraham and Lorentz attempted to account for Kaufmann's results in terms of the dynamics of the electron, Planck elaborated the relativistic response in terms of the dynamics of black-body radiation. This difference can be understood by taking into account what these different systems embodied. The electron carried the promise of a complete electromagnetic theory of matter, and hence it was the preferred object of study for the electromagnetic approach. Black-body radiation, on the other hand, carried the promise of a general theory bringing together all kinds of physical laws in a general theory. As such, we see how the choice of the preferred object of study aligns with the general aims that these different approaches tried to impose on the practice of fundamental physics at the time.

At the same time, however, we should not take Planck's general dynamics to establish, on the basis of theoretical considerations, that the velocitydependence of mass is merely an issue of definition. Planck has rather shown this on the assumption that the principle of relativity applies to the whole of physics. And, as we have seen, it is this assumption that is exactly at stake, at least according to Abraham, Ehrenfest and Kaufmann. As such, Planck's work should not be seen as a definite rebuttal of the challenge posed by the experiments, but rather as a relativistic alternative to the electromagnetic interpretation of Kaufmann's results. To understand how the relativistic interpretation came to be seen as the only viable one, we will now turn to the ways in which Einstein and Planck attempted to incorporate aspects of the electromagnetic program within the theory of relativity. 
Einstein and the Constitution of Radiation. In his (1909a) paper, Einstein turns to what he calls "an extremely important problem": the constitution of radiation (1909a, p. 185). This issue arises, he claims, because the Maxwell equations for empty space in themselves "do not say anything" (Einstein, 1909a, p. 185): they only offer an intermediary construct, which needs to be supplemented by other claims in order to say anything about the phenomena. The issue is a pressing one, he continues, because many of the current attempts to address it - Einstein discusses work by James Jeans, Lorentz, and Walther Ritz - do not provide satisfactory solutions, since they do not fit the facts. And while we have a radiation law that does fit all known facts, the theoretical foundations on which Planck built his distribution law are, according to Einstein, incompatible with this law (1909a, p. 186 - 188). Einstein is therefore brought to reconsider the theoretical assumptions underlying the study of radiation phenomena, and for this he turns to his quantum-hypothesis, which he had elaborated earlier in his (1905c; 1906b; 1907d; 1907f). ${ }^{54}$ Here he presents it as follows:

\footnotetext{
${ }^{54}$ It was this work on the quantum that Einstein described as "sehr revolutionär", in a letter to his good friend Conrad Habicht in which he discussed all the work he was carrying out in his annus mirabulis, 1905 (Klein et al., 1993, Doc. 27, p. 31 - 32). It showed him, as we have seen earlier, the limitations of any approach based on the Maxwell equations (see page 29 and Miller (1981, p. 133)). There is by now quite some extensive literature on the early stages of the quantum, especially regarding the interpretation of Planck's (1901a; 1901b) articles, which has even spawned a kind of meta-investigation on how historians have studied it: see e.g. the work by Martin J. Klein (1962; 1979), Thomas Kuhn (1978), Olivier Darrigol (2001), Clayton Gearheart (2002), Suman Seth (2010), and the references therein. For a historical discussion of Einstein's (1909a; 1909b) work, see
} 
A structure that is capable of carrying out oscillations with the frequency $\nu$, and which, due to its possession of an electric charge, is capable of converting radiation energy into energy of matter and vice versa, cannot assume oscillation states of any arbitrary energy, but rather only such oscillation states whose energy is a multiple of $h \cdot \nu$. Here $h$ is the constant so designated by Planck, which appears in his radiation equation. (Einstein, 1909a, p. 188)

The central conclusion of Einstein's quantum-work, as he shows by means of a discussion of his interpretation of Planck's distribution law (1909a, p. 188 - 190), was that Planck's constant $h$ was independent of the particulars of the physical systems studied by Planck in its derivation, and that it in fact pointed towards the existence of a discrete entity, the quantum of energy. The hypothesis that oscillation energy can only occur in quanta, expressed as multiples of $h$ and $\nu$, does not just concern the amount of radiation emitted and absorbed, but rather the constitution of radiation itself: it is "as if radiation consisted of quanta of the indicated magnitude" (1909a, p. 191). After a discussion of the experimental implications of his quantum-work, Einstein then concludes his paper with a sketch of what a theory of the constitution of radiation would look like.

For this, he turns to a discussion of how such a theory should treat a closed space containing an ideal gas, radiation, and ions that mediate an energy exchange between the radiation and the gas. Elaborating this then allows him to express the four quantities required to treat this case (the mean energy $\eta$ of a molecular structure; the light velocity $c$; the electric charge $\epsilon$;

Russel McCormmach (1970). 
and the frequency $\nu$ ) in terms of the fundamental constants that figure in Planck's distribution law (1909a, p. 192). The most important consequence of this result, Einstein then points out, is that the equation he obtains for Planck's constant, $h=\epsilon^{2} / c$, relates the light quantum constant $h$ to the electric charge $\epsilon$ (Einstein calls it the elementary quantum of electricity). This is a very significant result, since it suggests to Einstein that a theory accounting for the quantum could equally well account for the electron's constitution:

The most important aspect of this derivation is that it relates the light quantum constant $h$ to the elementary quantum $\epsilon$ of electricity. We should remember that the elementary quantum $\epsilon$ is an outsider in Maxwell-Lorentz electrodynamics. ${ }^{55}$ Outside forces must be enlisted in order to construct the electron in the theory; usually, one introduces a rigid framework to prevent the electron's electrical masses from flying apart under the influence of their electric interaction. The relation $h=\epsilon^{2} / c$ seems to me to indicate that the same modification of the theory that will contain the elementary quantum $\epsilon$ as a consequence will also contain the quantum structure of radiation as a consequence. (Einstein, 1909a, p. $192-193$ )

This now leads Einstein to a lists of constraints on the equation (or system of equations) that would constitute such a theory, one of which is that

\footnotetext{
${ }^{55}$ Here, Einstein refers to an article by Tullio Levi-Civita (1907), which, according to the editors of the Einstein papers, presented "a solution of the field equations corresponding to the motion of a stable, isolated charge moving at the speed of light" (1989, p. 553; note $67)$.
} 
the equation should transform into itself under application of the Lorentz transformation. And while such an equation is not yet available, Einstein concludes, we should not despair, since there do not seem to be that many possible candidates (1909a, p. 193).

As such, considerations pertaining to radiation phenomena were central to Einstein's elaboration of a relativistic response to the issues raised by the Kaufmann experiments, as they were for Planck (see page 46). More specifically, Einstein's quantum-work led him to an outline for a theory of the electron's constitution, because it indicated that the study of the constitutive entities of radiation and electricity should not focus primarily on the Maxwell equations: these only offer an intermediary structure ("eine Zwischenkonstruktion") that in itself does not say anything about the phenomena (1909a, p. 185), which entails that the electric elementary quantum $\epsilon$ is a stranger ("ein Fremdling") to the Maxwell-Lorentz electrodynamics (1909a, p. 192). On such a view, the Maxwell equations should therefore no longer be taken to inform us directly about any fundamental domain, as the electromagnetic program assumed. In this way, we thus see how the result of Einstein's (1909a) work on radiation and the quantum, i.e. the relationship between the light quantum $h$ and the electric charge $\epsilon$, allows him to incorporate the central aim of the electromagnetic program - to investigate the nature and constitution of charge - within the framework of relativity. It also provided him with a way to address the issue raised by Ehrenfest concerning the dynamics of the electron. At the same time, however, Einstein also generalizes the issue: the relativistic dynamics of the rigid body required to address Ehrenfest's issue no longer serves solely to account for the elec- 
tron, but equally well for the quantum. In this way, Einstein thus transforms Ehrenfest's issue: from a direct challenge to the theory of relativity, he is able to incorporate it within the relativistic research program.

Experiment and Historiography. It is important to stress here, however, that this incorporation was not carried through solely on the basis of theoretical considerations, as Janssen claims (2009, p. 35). Instead, it seems that at least two other factors - experimental and historiographical considerations- also played an important role, as I will try to argue here. ${ }^{56}$ Regarding the experimental factors, we have already seen that it was only because of Planck's thorough-going analysis of the material and conceptual machinery underlying Kaufmann's experimental set-up that the experimental challenge posed to the principle of relativity was defused. That experiment also played an important role for Einstein, is shown by the fact that he often employed radiation phenomena to develop possible experimental tests of the principle of relativity: in his (1906c), for example, he proposes an experiment on cathode rays ${ }^{57}$ in order to test what he calls the Lorentz-Einstein prediction of the velocity-dependence of mass against Abraham's and Bucherer's; and his short discussion of the Kaufmann experiments in his review article leads him

\footnotetext{
${ }^{56}$ Compared to the discussion before, these points will be rather schematic and short, because there is less direct material available to build them. In combination with my discussion of these aspects in Minkowski's and Laue's work, however, I do believe that they point to something significant.

${ }^{57}$ The generation of cathode rays is one of the first phenomena that, according to Einstein, required an account in terms of the quantum: he discussed these already in his first quantum paper, (1905c), as posing problems for wave-approaches.
} 
to the claim that more experimentation on both cathode and Becquerel-rays is needed to investigate whether the foundations of relativity are in correspondence with the observable facts (1907c, p. 439). ${ }^{58}$

Radiation phenomena, moreover, did not solely offer a domain to test particular consequences of the theory of relativity: they also offered the opportunity to test what Einstein considered to be the physical core of the theory, i.e. its claims about rods, clocks and light signals. This shows itself in Einstein's (1907e) article, in which he discusses the possibility of a new test of the relativity principle. His starting point here is an article by Johannes Stark (1906) in which he showed, by investigating the Doppler effect in canal

\footnotetext{
${ }^{58}$ This is the only place where Einstein explicitly discusses the Kaufmann experiments: even in his (1906c) proposal for alternative experiments, he does not refer to them. In $\S 10$ of this review article, Einstein discusses possible experimental tests for theories concerned with the motion of (charged) material point particles. After outlining how such theories could be tested by means of cathode rays, he then discusses Kaufmann's experiments on Becquerel rays: he presents the material set-up employed, and then reproduces the curve with the relativistic results. (In contrast to Kaufmann's original curve, Einstein does not reproduce Abraham's and Bucherer's results.) After referring to Planck's (1906b) re-analysis of the experiments, which has shown, according to Einstein, that Kaufmann's calculations are error-free, he then states that the systematic deviations of the theory of relativity from the results can be the consequence of either a not-yet-discovered source of error, or of a mismatch between the theory's foundations and observation, and that only more observations can decide this question. After admitting that Abraham and Bucherer scored better with regards to this particular experiment, he then states, however, that we should not ascribe them too high a probability, since their basic assumptions concerning the electron do not fit well with theories that concern a broader scope of phenomena (1907c, p. $436-439$ ).
} 
rays, that the positive ions in motion constituting these rays send out line spectra. Stark also attempted to carry out second-order measurements of this effect, but according to Einstein his experimental set-up was not adequately suited for this. Einstein then argues that the fundamental principles of the theory of relativity - the principles of relativity and of the constancy of the velocity of light - in fact predict this effect, and that this entails a formula which differs significantly from Stark's results. As such, carrying out Stark's experiments in a more precise way would allow us to test the principle of relativity. Of primary importance here, is that Einstein does not take this experiment to be concerned with a particular consequence of the theory, but with the principle itself, as the title of the (1907e) paper indicates. This becomes clear when Einstein returns to these results in his review article, and presents the oscillating ions studied by Stark as an illustration of the relativistic behavior of clocks (1907c, p. 422). ${ }^{59}$ In this way, we see how, for Einstein, experimental considerations pertaining to radiation phenomena played an important role in the elaboration of the theory of relativity as an alternative to the electromagnetic program.

Besides such experimental considerations, historiographical factors also

\footnotetext{
${ }^{59}$ Encouraged by Einstein (see the letter from Einstein to Stark on the 13th of April 1907 (Klein et al., 1993, Doc. 45, p. 47)), Stark continued this reseach. This led him to claim, in his (1907) article, that the phenomenon provided the first experimental confirmation of the quantum-hypothesis underlying Planck's radiation law. See note 5 to Einstein's letter to Jakob Laub, in which he describes Stark's application of the quantum-theory as very important ("sehr wichtig"), for an overview of historical work on Stark's claim (Klein et al., 1993, Doc. 125, p. 144).
} 
played an important role. This becomes clear, for example, in the addresses Planck (1908b) and Einstein (1909b) gave to the Naturforscherversammlung, the annual meeting of the German physics community. In these talks, both Planck and Einstein constructed what Staley calls research histories, i.e. "accounts of the past $[. .$.$] that scientists offer in key papers and re-$ view studies[, which] play a substantive role in shaping understandings of new theory" (2008, p. 294; original emphasis). ${ }^{60}$ What is significant about these research histories is that they both sketch a historical progression, from issues pertaining to electrodynamical and radiation phenomena towards the principle of relativity, that no longer presents the electromagnetic program as a challenge to the theory of relativity, but rather as part of the development towards it.

Planck's (1908b) talk was concerned with whether it was possible to formulate a unified concept of momentum that covers all the different domains of his general dynamics, just as the principle of action and reaction - which essentially, for Planck, concerns the conservation of momentum - did for Newtonian mechanics. This question first became pressing, according to Planck, when Lorentz's electrodynamics employed an ether-concept that denied the principle its general validity. An account that corrected this situation was then presented by Abraham, who showed that the generality of the principle could be saved, if one introduced an electromagnetic notion of momentum

\footnotetext{
${ }^{60}$ These are not the only research histories constructed for the theory of relativity. Chapter 8 of Staley's book (2008, p. 294 - 343) offers an extensive discussion of how Einstein, Planck and others throughout this period crafted the history of the theory of relativity in their work.
} 
besides the mechanical one. Abraham did this by means of an analogy: just as the principle of the conservation of energy can only be upheld when one takes into account electromagnetic energy, so one can only uphold the principle of action and reaction by introducing the concept of electromagnetic momentum. There is one problem, however, with Abraham's analogy between energy and momentum, according to Planck: there were already many different conceptions of energy at the time, which entails that the introduction of a new one did not constitute any radical change; with regards to momentum, however, we only had one conception, the mechanical one, which means that just introducing a second one entails a radical loss of simplicity and the introduction of substantial complexity. This then urges Planck to introduce his central question regarding a unified conception of momentum, and to show how the principle of relativity offers an adequate answer to it in terms of what he calls the law of the inertia of energy (1908b, p. $828-$ $830) \cdot{ }^{61}$

The central point here about the historical lineage drawn by Planck concerns the way in which he is able to present Abraham's work on electromagnetic momentum and energy as part of the development towards his relativistic solution to the problem posed by Lorentz's electrodynamics. In this way, Planck puts to the side the fact that Abraham proposed his notions in the context of the electromagnetic program: he did not intend them as additions, but rather as fundamental replacements for the mechanical concepts.

\footnotetext{
${ }^{61}$ In short, this law states that "the effect of forces acting on a body [is] transmitted by a momentum density whose source [is] a flow of energy" (Miller, 1981, p. 366). Miller there also gives a short overview of the rest of Planck's (1908b) paper.
} 
His historiography also allows Planck to set aside the fact that Abraham's electromagnetic notions, in the context of the Kaufmann experiments, actually posed a challenge for the use of the principle of relativity as the guiding principle for the foundations of physics. As such, Planck's history turns Abraham's electromagnetic theory into a predecessor of the central insight provided by the theory of relativity, i.e. the inertia of energy. In doing so, the electromagnetic program is no longer presented as a challenger to the principle of relativity, but rather as a step towards the real solution, the relativistic one.

Content-wise, Einstein's (1909b) talk is more or less the same as his (1909a) article: his topic is the constitution of radiation, and he addresses this issue by means of the suggestion that a theory accounting for both the electric charge and the light quantum should be developed. What is significant here, however, is that he proceeds by constructing a common history for the quantum, the electron, and the principles of the theory of relativity. His starting point for this are the first wave-conceptions of light, which gave rise to the development of the ether-concept, later employed by Maxwell in his electromagnetic theory. He then turns to the Michelson-Morley experiment, and Lorentz's treatment of it, which leads him to argue that it was unsatisfactory, since it did not grasp the central insight of experiment: the principle of relativity (1909b, p. 819). Einstein then highlights one particular consequence of the theory of relativity, namely that a body's inertial mass decreases by $L / c^{2}$ when it emits the radiation energy $L$. The importance of this relativistic consequence, Einstein claims, is that it leads to significant modifications for the basic ideas ("Grundanschauungen") of physics: it 
indicates that light has particular corpuscularian aspects (1909b, p. 820). However, because the theory of relativity in itself cannot offer us a theory of the constitution of light and radiation, Einstein turns to a discussion of his quantum-hypothesis, which proceeds along very similar lines as his (1909a) article, namely towards the suggestion of a theory that would account for both the corpuscularian and wave-aspects of light.

As such, Einstein's historiography draws a straight line from issues with wave-conceptions of light towards relativity and the quantum, which both, in their own way, point towards corpuscularian aspects of light. A first significant point about this is that the historical progression is no longer one from investigations into electromagnetic radiation towards the electron, as Kaufmann presented it in his Naturforscherversammlung lecture (1901b); this historical trajectory rather becomes part of the historical line from research on electromagnetic light phenomena to the principle of relativity and the quantum. A second point is that Einstein presents this historical progression as very much a shared history and future: as his title already indicates, his talk is concerned with the development of our ideas ("unserer Anschauungen") on the constitution of radiation, where this 'our', given the occasion at which Einstein gave his talk, probably is supposed to refer to the whole German physics community; and the next big thing in theoretical physics, according to Einstein, will be the development of the wave-and-corpuscletheory he suggests at the end (1909b, p. 817). As such, we see how historiographical considerations also played a significant role in the establishment of the theory of relativity as the only viable approach to fundamental physics, and of the electromagnetic worldview as something that had functioned as a 
stepping stone towards the theory of relativity, but now formed a world view in decline, as Helge Kragh calls it (1999, p. 114).

We thus see how, for both Planck and Einstein, their work on radiation phenomena was closely intertwined with their work on the theory of relativity, and this on different levels: theoretical, experimental and historiographical. This close connection now provides a good argument against Janssen's claim (i) that the (1905a) relativistic derivation of the velocity-dependence of mass shows that the phenomenon is purely kinematical, and that the relativistic study of this phenomenon is completely independent of any dynamical considerations pertaining to the systems displaying such behaviour. For it shows that both Einstein and Planck took the consequences of the theory to be in need of a theoretical elaboration, one which they thought would be provided in terms of the quantum. This also shows, again, how Planck's and Einstein's employment of the principles of the theory of relativity was essentially heuristic in nature. Both employ the principles by applying them to particular laws that are suppposed to govern openstanding problems or phenomena of interest, which leads them to new possible claims regarding these phenomena. These relativistic claims then in turn function as the starting point for further investigations of the underlying physical structure that brings about these phenomena.

This close connection also provides a good argument against Janssen's claim (ii) that it were theoretical considerations that established the theory of relativity as a replacement for the electromagnetic world view. For it shows that both experimental and historiographical considerations pertaining to radiation phenomena and their relation to relativity and the electron also 
played an important role in the elaboration of the theory of relativity as an alternative for the electromagnetic program: it was by incorporating the study of the electron into the relativistic program that Einstein and Planck were able to defuse the electromagnetic challenge.

In the following section, this approach to the theory of relativity, with its heuristic conception of the theory's principles, and its experimental and historiographical relation to radiation phenomena, will be contrasted with the Minkowskian approach. This will then allow me, in a historical way, to address both Janssen's particular claim that it was Laue's work on Ehrenfest's issue that definitely showed the kinematical nature of the velocitydependence of mass, and his general claim (iii) that this shows that Minkowskian framework provides the natural interpretation for the theory of relativity (Janssen, 2009, p. 39). That such a distinction between these two approaches can, and needs to be, drawn, is already suggested by the fact that different physicists at the time saw themselves required to point out differences between Einstein's theory of relativity and Minkowski's theory. One of them was Einstein himself, who published a paper, together with Laub, in which they argue that Minkowski's electromagnetic treatment of the ponderomotive force exercised on a moving body is in conflict with what they call electron theory (1908b, p. 541) ${ }^{62}$ Another scientists drawing such a distinction was Abraham, who in (1909) pointed out that Minkowksi's framework differed from Einstein's and Planck's with regards to the issue of whether bodies have a constant or a variable rest mass, a claim he repeated in Italian in (1910).

\footnotetext{
${ }^{62}$ Lewis Pyenson (1985, p. $81-82$ ) offers a short discussion of Einstein's work with Laub on Minkowski.
} 


\subsection{Minkowski and Laue's Normative Interpretation}

The Minkowskian Universalization of Relativity. The central aim of Minkowski's work on relativity, as he states it in his first presentation on the topic to the Göttinger Mathematischen Gesellschaft (1907), is to show how the theory offers mathematicians the opportunity to contribute to actual physical research. The theory allows for this, according to Minkowski, because it entails that the physical world is a well-known mathematical object: a fourdimensional non-euclidean manifold, an object with which mathematicians already have quite some experience (1907, p. 927).

In his well-known Grundgleichungen article (1908), ${ }^{63}$ Minkowski then elaborates the specifics of this mathematical-physical program. In order to do this, Minkowski first introduces a three-fold distinction between the theorem, the postulate and the principle of relativity. The theorem comes down to the mathematical fact that the Maxwell equations are invariant under the Lorentz-transformations. The postulate is the hypothesis that as-of-yet unknown laws concerning material bodies will also display such a covariance under the same transformations. The principle, finally, expresses the belief that the introduced covariance will hold as a determinate relation between observable quantifiable characteristics of moving bodies (Minkowski, 1908, p. 54).

On the basis of this, Minkowski then proceeds as follows. In the first section, he outlines his fundamental equations describing the electromagnetic ether, void of any material systems, under the rule of the theorem of relativity.

\footnotetext{
${ }^{63}$ This is the only work by Minkowski on relativity that was published during his life. He died of appendicitis in 1909.
} 
The second section is then concerned with the discussion of electromagnetic processes in moving material systems. In order to discuss these, he introduces three axioms: first, that a body at rest can be described by the laws of electrostatics; second, that the speed with which light propagates through empty space forms an upper limit on the motion of all physical systems; and third, his principle of relativity, i.e. the claim that Lorentz covariance holds as a determinate relation between observable quantities for moving bodies (Minkowski, 1908, p. 72). On the basis of this, he then formulates his fundamental equations for the electrodynamics of moving bodies.

The physico-mathematical framework he has developed on the basis of these three axioms then allows him, more specifically, to employ the principle of relativity as a normative instrument to evaluate electrodynamical theories, which he showcases for the theories of Emil Cohn (1901) and Lorentz (1904). What he does is to compare these theories' fundamental equations with the ones he has obtained for the electrodynamics of moving bodies. Given that the Minkowskian equations are based on his three axioms, which are to ensure the equations' covariance with respect to the Lorentz transformations, they do indeed offer a way to evaluate whether the equations of other theories are also relativistic. In this way, it becomes clear how Minkowski sees the participation of mathematicians in physical research.

Moreover, Minkowski does not confine the evaluatory normativity of the principle of relativity merely to the electrodynamics of moving bodies. In the appendix of his Grundgleichungen paper he turns to classical mechanics. Many authors, he claims, consider this domain to be in contradiction with the principle of relativity, a conflict that arises, according to Minkowski's frame- 
work, from the fact that the equations of mechanics and the electrodynamic equations are both covariant with respect to the expression $-x^{2}-y^{2}-z^{2}+c^{2} t^{2}$, but for different determinations of $c$ : for mechanics, $c=\infty$, whereas for electrodynamics it is a finite, determinate quantity (1908, p. 99). However, this situation can be overcome, according to Minkowski, if we follow his mathematical machinery, which tells us that we should reformulate the theory of mechanics in terms of the principle of conservation of energy (1908, p. 108 - 109). ${ }^{64}$ We thus see how Minkowski's principle of relativity functions as an evaluative and normative constraint on the formulation of any physical theory, whether it be Lorentz's or Cohn's electrodynamics or Newtonian mechanics.

This point marks one central difference between Minkowski's and Einstein's approach to the theory of relativity. For Einstein, the principles of the theory primarily functioned as a heuristic instrument: they primarily served as a way to open up new areas of physical research. For Minkowski, on the other hand, the principle served as a norm constraining the practice of physical theorizing: any theory should conform to the principle of relativity, and if a theory does not, the principle of relativity tells us how to change

\footnotetext{
${ }^{64}$ In the introduction to his article, Minkowski describes this as a very surprising consequence ("einem sehr überraschenden Erfolge") of his theory (1908, p. 56). This seems to suggest that Minkowski's aim was to present his theory as something more than just a mathematical reformulation of relativity theory, because it led to new insights. In a sense, this claim of originality by Minkowski here is a bit surprising and quite unfair, because in his (1907) lecture he had also hinted at this consequence, but there he admitted that it was first presented by Planck (1908a).
} 
it. ${ }^{65}$ This becomes especially clear in the lecture Minkowski presented to the Naturforscherversammlung, titled Raum und Zeit (1909), in which he elaborates the physical content of his interpretation of the theory of relativity. The theory's central claim, according to Minkowski, is that the laws of nature are invariant with respect to the transformation group $G_{C}$, where $c$ is the velocity of light, rather than with respect to the transformation group $G_{\infty}$ of Newtonian mechanics, where $c=\infty$. Expressed in different terms, this comes down to the claim that the collection of admissible reference systems is constrained by the $G_{C}$-invariance of the laws of physics: one can transform the reference system employed by means of the group $G_{C}$ without thereby changing the form of the laws of physics (Minkowski, 1909, p. 78).

What the mathematical formalism of the transformation group $G_{C}$ captures is that our three-dimensional reference frames for space and time are constructed by us within four-dimensional space-time. In this way, the formalism shows, according to Minkowski, that the principle of relativity goes further than Einstein thought: Einstein only relativized time, and it took the boldness of mathematical culture ("Verwegenheit mathematischer Kultur")

\footnotetext{
${ }^{65}$ This is not to say that Einstein's use of the principle was exclusively heuristic, while Minkowski's was exclusively normative, but rather that there is a difference in emphasis: while Einstein also sometimes formulates the principles in normative terms, he primarily employs them as heuristic instruments that guide the development of the theory; and while for Minkowski the principles can also act as guides, he primarily uses them to evaluate other theories. As will be discussed in section 4 in more detail, the most important difference between the two approaches is not, however, that they use the principles as either guides or constraints, but rather their different epistemological characterizations of the principles.
} 
to see that both space in itself and time in itself, according to the principle, had to be relegated to the shadows. For this reason, Minkowski proposes to stop using the term 'postulate of relativity' to refer to the $G_{C}$-invariance of the laws of physics, since he finds it rather bland ("sehr matt"). Instead, he proposes, we should speak about what he calls the "postulate of the absolute world', because it better expresses the true meaning of the postulate of relativity:

Since the postulate comes to mean that spatio-temporal phenomena manifest themselves only in terms of the four-dimensional world, but the projection in space and time may still be performed with certain liberty, I prefer to call it the postulate of the absolute world (or briefly, the world-postulate). ((Minkowski, 1909, p. 82), translated in (Corry, 1997, p. $296-297))$

And the central insight of what Minkowski calls the electromagnetic program started by Lorentz and continued by Einstein is that this world-postulate has exceptionless validity ("ausnahmslose Gültigkeit") (1909, p. 88). This indicates a significant difference with Einstein's and Planck's approach to the theory of relativity: for them, the theory's range of validity was still an open question, which was to be decided on an experimental basis. Another difference concerns the theory's domain, and the way in which it was to be applied. For Einstein, the principles only applied to rods, clocks and light signals, and it could only be applied in a piece-meal way to specific cases, since a relativistic world view was still lacking. Minkowski, on the other hand universalizes the domain: the theory's principles now apply to the observable characteristics of all moving systems in general as they manifest themselves 
in the four-dimensional non-Euclidean manifold that is the world. In this way, the theory's principles come to function as norms for all present and future theories: from now on, physical theories are only concerned with the behavior of systems that can be described in terms of $G_{C}$-invariant laws.

One particular consequence of this ascription of unlimited validity to the postulate of the absolute world now is that it directly entails the dismissal of any real challenge posed to the principle of relativity by the Kaufmann experiments. For the universality of Minkowski's postulate entails that it does not make any sense, within a $G_{C}$-invariant mechanics, to talk about a rigid body, for in that case it would be possible to detect some kind of ether-drift (1909, p. 80). ${ }^{66}$ In this way, the Minkowskian formalism thus rules out, by means of its universalization of the principle of relativity, that the Kaufmann experiments can pose a challenge to the principle, since the experiments were precisely concerned with the issue of whether the electron was rigid or deformable. In fact, Minkowski's move goes even further. Given that, on his view, the postulate of the absolute world underlies any experience of space and time, it follows that the postulate itself is not open for experimental investigation or testing in general: there does not seem to be any direct way to test experimentally whether the actual world does indeed obey the postulate of the absolute world.

This clearly shows that Minkowski's approach to the theory of relativ-

\footnotetext{
${ }^{66}$ Ehrenfest (1909) made a similar claim: he showed in an intuitive way that, following Minkowski's ideas, the notion of a rigid body leads to two contradictory claims with regards to a rotating cylinder. Miller (1981, p. 235 - 257) and Staley (2008, p. 284 - 291) offer a broad overview of the discussions on the rigid body during this period.
} 
ity differs from Einstein's. On Einstein's view, as we have seen earlier (see page 53), there was the possibility of experimental testing, not only of consequences of the theory such as the formulae for the velocity-dependence of the electron's mass, but also of the theory's physical core, i.e. its physical interpretation of time in terms of the oscillating ions constituting canal rays. The two approaches differ not only with regards to experiment, however, but also concerning their historiography. For Planck and Einstein, as we have seen (page 54), the theory of relativity primarily emerged out of issues pertaining to radiation phenomena. For Minkowski, on the other hand, the principle of relativity's physical importance primarily emerged out of issues regarding classical mechanics. For while he admits that it was the Maxwell equations that led to the theorem of relativity - i.e. the mathematical fact that the laws of electrodynamics are Lorentz invariant -, the real physical significance is that this new invariance-conception leads us to the insight that the equations of mechanics are only approximations of reality (Minkowski (1907, p. 935); Minkowski (1908, p. 99); Minkowski (1909, p. 78)), since it is this insight that leads Minkowski to his postulate of the absolute world and its reconceptualization of space and time.

In this way, it becomes clear how the Minkowskian normative approach to the theory of relativity differs from the Einsteinian heuristic approach. For Minkoski, the postulate of the absolute world applies to the observable characteristics of all moving systems within the non-Euclidean four-dimensional manifold that is the world, which entails the following: it functions as a norm for the formulation and evaluation of all physical theories; it is prior to any possible physical experiment; it therefore has exceptionless validity; and it 
emerged out of issues pertaining to mechanical phenomena. For Einstein, on the other hand, the theory of relativity applies to the physical behaviour of rigid rods, clocks and light signals, which entails the following: it serves as a heuristic tool in the investigation of new domains, and has to be applied in a piece-meal way; it can be tested experimentally; the range of its validity is therefore still an open question; and it emerged out of issues pertaining to radiation phenomena.

Laue replies to Ehrenfest. That Minkowski's universalization of the principle of relativity dismisses, by means of a priori postulation, the challenge posed by the Kaufmann experiments, shows itself in a subtle way in Laue's (1911a) article. According to Janssen (2009, p. 35), this work clearly showed the kinematical nature of the velocity-dependence of mass (see page 18). Laue starts his article by pointing out that, on the assumption that Newtonian dynamics forms a limiting case for infinitely low velocities, Einstein (1905a) and Planck (1906a) already sorted out the relativistic dynamics of the mass point. On this basis, they then obtained the equations for the velocitydependence of longitudinal and transverse mass, now confirmed by several unnamed electron-experiments. At the same time, however, there are still unresolved dynamical issues, one of which was raised by Ehrenfest (1907). This issue, according to Laue, concerned the question of whether this dynamics of the mass-point also holds, when one conceives of the form of the deformable electron not in terms of radial symmetry, as one normally does, but rather as a kind of ellipsoidal form (Laue, 1911a, p. 524).

Einstein (1907a), according to Laue, already responded to Ehrenfest's question in the affirmative. Given that this response lacked in generality, 
Laue returns to the issue, but he proposes to proceed, however, in the opposite direction. While Einstein and Planck started with the dynamics of the mass point, Laue, on the other hand, will start from his relativistic continuum dynamics, firmly based within the Minkowskian framework, as is clear from the formulation of the principle of relativity he presents in his (1911b): ${ }^{67}$

The principle of relativity claims: through ever increasing approximations one can determine, out of the the totality of natural phenomena, ever more precisely a reference system $x, y, z, t$, in which the laws of nature hold in a precise and mathematically simple form. This reference system is not, however, precisely set by the phenomena. There rather is a threefold infinite variety of equal systems, which move with uniform velocities with respect to each other. (Laue, 1911b, p. 33 ; personal translation)

Proceeding in this way then allows Laue to show how one can obtain equations for the rest mass of a completely static system and for the longitudinal and transverse mass of such a system undergoing quasi-stationary acceleration, independent of the system's precise form (1911a, p. $539-541) .{ }^{68}$ Laue then states that the electron with its field forms such a system, which leads

\footnotetext{
${ }^{67}$ Laue's (1911b) was, as Staley put it, "[t]he first and authoritative textbook on relativity" (2008, p. 334). There he gives two reasons for starting with a dynamics of the continuum, instead of a dynamics of the mass point: because otherwise it would not be possible to account for Ehrenfest's question and the Trouton-Noble experiment (see Janssen $(2009$, p. $41-47)$ for a discussion of this experiment and Laue's work on it); and because it would require more hypotheses than strictly needed (Laue, 1911b, p. 148).

${ }^{68} \mathrm{~A}$ completely static system, on Laue's account, is a system that, in its rest frame, is in static equilibrium, without interacting with other bodies (1911a, p. 539). A sys-
} 
him to the claim that whatever its form, it must conform to the dynamics of the mass point when it is undergoing quasi-stationary acceleration. As such, it is not possible, on the basis of these results, to investigate further into the electron's form, its charge-distribution, or the question of whether the electron's constitution is purely electromagnetic, and Einstein therefore responded correctly to Ehrenfest's question (Laue, 1911a, p. 541 - 542).

In this way, it becomes clear that Laue's response does not completely provide a satisfying answer to Ehrenfest's question, since they are in fact concerned with different kinds of questions. For Laue, the central issue was whether the dynamics of Einstein's mass point electron would remain the same if one ascribed a different form to the electron. This was not, however, Ehrenfest's central concern: for him, the question was not about the specific form of the deformable electron, but rather the stability of a deformable electron in general. Both Ehrenfest's paper and Kaufmann's experiments essentially concerned the question of whether the electron is rigid (Abraham) or deformable (Lorentz-Einstein, Bucherer). Ehrenfest is quite explicit about this: in the statement of his question, he points out that the issue arrises independently of the precise form of the deformable electron, by referring to a claim made by Planck (1906a, p. 137) (see page 24). This is also clear from the fact that he raises the same issue for Bucherer's deformable electron:

tem undergoing quasi-stationary acceleration is a system that is characterized by such an acceleration that what he calls its inner state ("innere Zustand"), characterized by the system's rest energy $E_{0}$ and its stress tensor $\mathbf{p}_{0}$, does not change noticeably (Laue, 1911a, p. 541). The motion of the electrons in Kaufmann's experiments was shown by Abraham (1903, p. 159) to be quasi-stationary. 
while it is clear, he claims there, how Abraham's rigid electron is stable, this is not the case for the deformable electron (Ehrenfest, 1906, p. 302). Moreover, Einstein also read the issue in these terms, as is clear from his response to Ehrenfest: there he points out that while the stability issue is easily solvable if one postulates the electron as a rigid body, a relativistic account of this is not available yet, since a dynamics of the rigid body is still lacking (1907a, p. 208). Einstein's claim there was not that the form of the deformable electron does not matter, but rather that the dynamics of such an electron is still an open question from a relativistic point of view, and one that he took to be important, it seems, given his work on the electron's constitution in his (1909a).

As such, Laue's (1911a) article should not be read as providing an actual answer to Ehrenfest's question. It only shows that, assuming the Minkowskian principle of relativity, we can obtain the relativistic equations for the velocitydependence of mass, regardless of what precise form we ascribe to the electron. What it does not show, however, is that the electron is in fact deformable rather than rigid. Laue's treatment of the issue rather makes clear that Ehrenfest's original concern, whether a deformable electron can actually be stable in comparison with a rigid body, does not make much sense anymore: the only question that remains is whether the form ascribed to the deformable electron makes a difference. If we therefore want to claim that Laue put aside the electromagnetic challenge raised by Kaufmann's experiments and Ehrenfest's question, then we have to recognize that that is not because he established the purely kinematical nature of the velocity-dependence of mass, but rather because the Minkowskian framework excludes, by means of 
its a priori universalization of the principle of relativity, the possibility of a relativistic rigid body.

We should therefore not read Laue's results as some kind of relativistic refutation of the electromagnetic program. Laue himself also did not see his work in this way, as is clear from the way he treats, for example, the dynamics of the electron (conceived in turn as a mass point, a charged sphere, an elastic body and as a completely static system) in his handbook (1911b, p. 162 - 170). He there points out that, while the principle of relativity is in agreement with experiments by Bucherer and Karl Erich Hupka on the velocity-dependence of mass, it is not yet completely without objections ("einwandfrei"). ${ }^{69}$ He then turns to a short discussion of the attempts to formulate a completely electromagnetic conception of the electron, after which he points out that, according to him, the principle of relativity seems to fare better than them: for while such conceptions are committed to a purely electromagnetic mass in order to account for the velocity-dependence of mass, relativity leaves room for many different possible conceptions of mass (Laue, 1911b, p. 167). ${ }^{70}$ As such, Laue indicates, a choice between the

\footnotetext{
${ }^{69}$ Bucherer $(1908)$ and Hupka $(1909 ; 1910)$ both ran experiments on the velocitydependence of the electron's mass with experimental set-ups that attempted to improve on Kaufmann's. Laue presents both experiments as providing results in favor of the theory of relativity. In both cases, however, there were severe criticisms: for a discussion of Bucherer's experiments and Adolf Bestelmeyer's criticism, see Miller (1981, p. 345 - 350) and Staley (2008, p. 250 - 254); for the experiments carried out by Hupka and criticized by Wilhelm Heil (both doctoral students of Planck), see Miller (1981, p. 376 - 377; note 9) and Pyenson (1985, p. $202-203)$.

${ }^{70}$ This claim in itself already constitutes a very interesting turn in the debate, since the
} 
two approaches with respect to this particular issue is still a real possibility, since the experiments carried out until now cannot decide for us.

In his concluding remarks, Laue then elaborates how he sees the more general relationship between the principle of relativity and the electromagnetic program. Here, the issue is not even one of a choice between the two. Rather, the electromagnetic program and the principle of relativity here concern two completely separate issues. According to Laue, the question of whether we should take the fact that all physical forces lead to behavior that is Lorentz invariant as an argument for a common, electromagnetic, origin, is one that is completely outside the concerns of the relativistic approach. The only thing we can conclude from this fact, Laue claims, is that the principle of relativity holds for any domain of physics, which leads him to characterize it as a criterion of admissibility for any physical theory (1911b, p. 185 - 186). As such, we see how Laue follows Minkowski in characterizing the principle of relativity as a normative constraint on the practice of physics, and that it is this that allows him to dismiss Kaufmann's experiments and Ehrenfest's question as a serious challenge to the principle of relativity, and to characterize the electromagnetic program as a completely detached issue. In this way, the Minkowskian framework in which Laue formulates his relativistic continuum dynamics does not so much allow him to definitely refute

fact that the theory of relativity did not provide a definite answer regarding the precise constitution of the electron was only a few years earlier taken as a big problem for the theory (see the discussion of Ehrenfest's paper in section 3.1, as well as Staley's discussion of challenges posed by the theory of the electron to the principle of relativity (2008, p. 260 $-293))$. 
the challenges raised by the electromagnetic program, but rather to put them aside as issues that are no longer pressing, since they are outside the concerns of the relativistic approach. This also shows the difference with Einstein's approach to relativity at the time, since he attempted, in his (1909a) work, to incorporate Ehrenfest's issue within the relativistic research program (see page 52).

\section{Concluding Remarks}

In the previous section I have shown that the historical narrative about Kaufmann's experiments underlying Janssen's (2009) philosophical claim that the theory of relativity establishes that the velocity-dependence of mass is a kinematical phenomenon about which nothing more can be learned, and that dynamical studies were therefore illusory and misguided, does not hold op against the historical development of the theory of relativity. My concern is thus not primarily with Janssen's philosophical claim about how to interpret the special theory of relativity, but rather with the three particular historical claims on which Janssen based his argument for this philosophical claim: (i) that Einstein's (1905a) derivation of the relativistic formulas for the velocity-dependence of the electron's longitudinal and transverse mass showed that he saw that the phenomenon is purely kinematical; (ii) that the acceptance of these formulas was mainly a result of theoretical considerations, and that experimental considerations played no real role of importance; and (iii) that the Minkowskian framework provides the natural interpretation of the theory of relativity, and that within this framework the kinematical nature of the phenomenon becomes especially clear, as Laue's (1911a) shows. 
In this concluding section, I will start with a discussion of these three claims, which will lead me to my claim that historically speaking, we can distinguish between a heuristic and a normative approach to the theory of special relativity. Given that Janssen explicitly states that his philosophy of physics is informed by the history of physics, I will then reflect a bit about what this could mean for Janssen's general philosophical claim that the theory of special relativity reveals the kinematical nature of phenomena such as the velocity-dependence of mass.

With regards to claim (i), sections $3.1,3.2$ and 3.3 have shown that dynamical considerations did play an important role in the development of a relativistic response to the issues raised by Kaufmann's experiments. It has been shown, more specifically, that Einstein took Ehrenfest's challenge to pose an actual issue for the theory of relativity, and that it urged him to investigate the implications of the principles of the theory of relativity for a dynamics of the rigid body. We have also seen that it was Planck's elaboration of a general dynamics, on the basis of his study of the dynamics of the black-body, that led to a relativistic account of the velocity-dependence of mass as a phenomenon of merely secondary importance. Finally, we have also seen that Einstein was actively searching for a response to Ehrenfest's question regarding the electron's constitution, through his work on radiation phenomena and the quantum-hypothesis. As such, it is not the case that Einstein (or Planck) considered the issues surrounding the velocity-dependence of mass to be merely kinematical in nature, or that they believed that a relativistic study of them would be independent of any dynamical considerations of the systems displaying such behaviour. 
Concerning claim (ii), it has turned out that experimental considerations did play an important role in the elaboration and establishment of the theory of relativity. We have seen, for example, that radiation phenomena, for Einstein, provided a way to experimentally test and elaborate both the central principles and the particular consequences of the theory. In the case of Planck as well, experimental considerations played an important role: it is only because of his thorough analysis of both the material and conceptual machinery of Kaufmann's experimental set-up that he was able to argue that the experimental results should not be taken as a definitive refutation of the principle of relativity. Moreover, many other early adherents of the relativistic approach also engaged quite profoundly with the experimental aspects of both Kaufmann's experiments and their implications for the theory of relativity: both Planck and Stark, for example, participated in a thorough exchange with Kaufmann about Kaufmann's material set-up and the validity of Planck's analysis; ${ }^{71}$ Bucherer $(1908)$ and Hupka $(1909 ; 1910)$ both performed experiments on the velocity-dependence of the electron's mass, which improved on particular aspects of Kaufmann's experiments; and Laub not only proposed, together with Einstein, a way to distinguish experimentally between

\footnotetext{
${ }^{71}$ Kaufmann (1907) discussed some particular aspects of Planck's analysis, to which both Planck (1907) and Stark (1908) responded, which in turn solicited a response by Kaufmann (1908). This discussion concerned the question of whether any residual gas in Kaufmann's experimental set-up could have been ionized by the radiation passing through it, which would influence the effective strength of the electric field applied, and which would thus have an influence on the exact deflection of the electrons constituting the radiation (Staley, 2008, p. $249-250$ ).
} 
Lorentz's electrodynamics and the theory of relativity (1908a), but he also published an extensive review article on the experimental foundations of the principle of relativity (1910). As such, this indicates that experimental considerations did play an important role, both regarding the particular issue of the velocity-dependence of mass and the more general elaboration of the theory of relativity. Moreover, it has become clear that the development of a relativistic response was not solely an issue of either theory or experiment, but equally well of historiography: it was through the integration of certain aspects of the electromagnetic program and of the study of radiation phenomena into a historical progression towards the theory of relativity that the theory could be presented as actually overcoming the issues and challenges raised by the electromagnetic program.

In this way, we immediately come to see that claim (iii) is problematic as well, since with regards to these issues - theory, experiment, and historiography - a significant distinction can be drawn between Einstein's and Minkowski's approach to the principle of relativity and the light principle. Einstein, at the time, took the principles of the theory of relativity primarily to function as a heuristic instrument, which in itself only made claims about rods, clocks, and light signals. In order to arrive at further claims, the principles had to be applied to the laws of physics, and this could result in new insights that could then be investigated further by other means. One particular aspect of this interpretation was that, because of Einstein's physical interpretation of the theory in terms of rods and clocks, not only the consequences of the application of the principles but also the principles itself were open to experimental investigation (see page 53). As such, the 
theory of relativity was, for Einstein, to be elaborated by means of both theoretical and experimental considerations, and this especially with regards to radiation phenomena. This preference for radiation phenomena seems to have had two reasons: first, because they embody a central aim of the theory of relativity, i.e. to bring together all kinds of laws of physics under a few guiding principles; and second, because they offered an essential object of study for the construction of an all-encompassing relativistic view of nature, since they showed the limitations of the Maxwell equations. As such, we also come to see why the theory of relativity, according to Einstein (1909a), had its historical origins in the study of radiation phenomena. That the principles of the theory, on this heuristic approach, were open to experimental investigation and testing also entailed that their range of validity was still an open question: as Einstein points out, for example, in his (1907c, p. 439) review article, experiments could lead to the conclusion that the principle of relativity does not completely agree with the facts. ${ }^{72}$ Finally, this also entailed that the theory of relativity had to be elaborated in a piecemeal way, as is clear from Einstein's remarks, in his (1907b) article, on the lack of a relativistic world view: this entailed that, at the time, the only way to elaborate the theory was by means of specific electrodynamical cases, since only there were the principles of the theory on secure ground.

For Minkowski, on the other hand, the principle primarily functions as a

\footnotetext{
${ }^{72}$ A similar view was held, according to Goldberg (1976, p. 136), by Kurd von Mosengeil, a student of Planck on whose work Planck based his relativistic dynamics (1908a): "[a]s far as the validity of the principle of relativity was concerned, that was still an open question" for Mosengeil.
} 
universal norm on the formulation of any physical theory, since it applies to the observable characteristics of any moving body: as Laue puts it, the principle acts as a criterion of admissibility for any physical theory (1911b, p. 185 - 186). The most important consequence of this conceptualization, according to Minkowski, is the relativization of both space and time, which become constructs of our making within the four-dimensional non-Euclidean manifold that is the physical world. This entails first, that the principle's validity becomes exceptionless, and second, that the principle is not open for experimental testing, since it precedes any possible physical experience. As such, the theory of relativity was to be elaborated, on Minkowski's view, primarily through the mathematical means offered by Minkowski's four-dimensional space-time geometry. The main reason for this mathematization of the theory of relativity seems to have been Minkowski's aim to show that mathematicians could contribute to the elaboration of fundamental physics: it puts them in a position to elaborate and evaluate other theories (such as Cohn's and Lorentz's, as we have seen on page 62), and in this way it provides evidence for what Minkowski calls a pre-established harmony between pure mathematics and physics ("eine prästabilierte Harmonie zwischen der reinen Mathematik und der Physik") (1909, p. 88). ${ }^{73}$ As such, we also come to see why Minkowski elaborated the real physical importance of the theory of relativity through its historical relation with Newtonian classical mechanics: showing how the group $G_{\infty}$ is an approximation of $G_{C}$ allows Minkowski to present his four-dimensional space-time geometry as embodying the new

\footnotetext{
${ }^{73}$ For a historical discussion of this concept of a pre-established harmony, see Pyenson's chapter 6 on this topic (1985, p. $137-157)$.
} 
kinematical basis for any physical theory and as replacing the Newtonian concepts of space and time.

These three points now allow me to address both Janssen's treatment of the Kaufmann episode in particular, and his philosophical claim that the theory of relativity shows the kinematical nature of relativistic phenomena in general. Let us first turn to the Kaufmann episode. We have seen that both Planck and Einstein attempted, on the basis of dynamical considerations pertaining to radiation phenomena, to develop a relativistic response to the issues raised by Kaufmann's experiments and Ehrenfest's paper. Planck's general dynamics, based on his study of black-body radiation, entailed that the velocity-dependence of mass is primarily an issue of definition from a relativistic point of view. And Einstein's work on the constitution of radiation led him to an outline for a relativistic theory that would account for the structure of both the electron and the quantum. As such, we see how the heuristic approach to the theory of relativity allowed for the possibility of elaborating a direct answer to the issues raised by Kaufmann and Ehrenfest: it is by the piecemeal application of the principles of the theory to the laws of physics that govern specific cases that additional statements can be derived, which are then to be investigated further by turning to the dynamics underlying those specific cases. On the normative approach to the theory, as we have seen in our discussion of Minkowski and Laue, this was not possible, since Minkowski's universalization of the principles entailed the exclusion of a relativistic study of the dynamics of the electron. On the basis of this, Laue then sidesteps the actual question raised by Ehrenfest, by showing that the precise form of the deformable electron does not make a 
difference, rather than actually addressing the issue of whether the electron is rigid or deformable. As such, we see that the Kaufmann episode does not provide historical evidence for Janssen's claim that the theory of relativity established the kinematical nature of the velocity-dependence of mass. The discussion rather showed that, on the heuristic approach, the development of a relativistic account of the velocity-dependence of mass was closely intertwined with reflections on the dynamics of particular systems displaying such behaviour, such as radiation and the electron. On the normative approach, on the other hand, we see that these dynamical issues are no longer of concern for the relativistic program.

In this way, we arrive at Janssen's general philosophical claim, that special relativity establishes the kinematical nature of relativistic phenomena, and that this becomes especially clear in its Minkowskian formulation, which forms the theory's natural interpretation. What the discussion above shows is that it is difficult, historically speaking, to talk about the natural interpretation of the theory. A first thing to notice is that the historical discussion indicates that it is difficult to speak of the theory of relativity at the time. We are rather dealing with different approaches to relativistic physics, each with their preferred objects of study, their particular ways of elaborating the theory, and their own historiography: on the heuristic approach, the elaboration of the theory proceeded in a piecemeal way by means of theoretical and experimental considerations pertaining to specific cases such as radiation phenomena, which entailed that the range of validity of the theory's principles was still an open question; on the normative approach, the elaboration of the theory proceeded through the mathematical study of four-dimensional 
non-Euclidean geometry, which entailed the exceptionless validity of the theory's principles. And the choice for a particular approach, it seems, was primarily made on the basis of what one took to be the central aim of the principle of relativity: for Planck and Einstein, it was to bring together all kinds of laws of physics, whereas for Minkowski it was to show that pure mathematics could contribute to fundamental physics.

As such, it is difficult to speak about the theory of relativity showing that certain phenomena have a particular nature, be it kinematical or dynamical. Instead, the nature of a particular phenomenon under study seems to depend on the approach employed to study it: on the heuristic approach, the velocity-dependence of mass was ultimately taken to be a phenomenon that had to be studied both kinematically and dynamically; on the normative approach, on the other hand, only the phenomenon's kinematics could be studied from a relativistic perspective. This suggests that phenomena of scientific interest do not have a particular nature which is described by a theory's natural interpretation. Instead, what a particular scientist takes to be a phenomenon's nature depends on his or her particular aims and interests, and a phenomenon's nature is therefore itself an issue of scientific debate and dispute, which shows itself in the way in which scientists characterize the theoretical, experimental and historiographical manifestations of the phenomenon. This does not mean that there are no phenomena, but rather that there are always different ways to approach a phenomenon, i.e. to turn it into a particular scientific object of study. ${ }^{74}$

\footnotetext{
${ }^{74}$ Because of this, I have not phrased my discussion of Einstein, Planck, Minkowski and von Laue in terms of the dynamics vs. kinematics distinction. Especially the heuristic
} 
Abraham, M. (1902). Dynamik des Elektrons. Nachrichten von der Gesellschaft der Wissenschaften zu Göttingen, MathematischPhysikalische Klasse: $20-41$.

Abraham, M. (1903). Prinzipien der Dynamik des Elektrons. Annalen der Physik, 10: $105-179$.

Abraham, M. (1904). Die Grundhypothesen der Elektronentheorie. Physikalische Zeitschrift, 18: 576 - 579.

Abraham, M. (1905). Theorie der Elektrizität. Zweiter Band: Elektromagnetischer Theorie der Strahlung. Teubner.

Abraham, M. (1909). Zur elektromagnetischen Mechanik. Physikalische Zeitschrift, 21: $737-741$.

Abraham, M. (1910). Sull'Elletrodinamica di Minkowski. Rendiconti del Circolo Matematico di Palermo, 30: 33 - 46.

approach does not seem to fit into the category of either kinematical or dynamical, since it seems to investigate the relativistic phenomena from both sides: while the theory, on the heuristic approach, is tied to particular systems (rods, clocks, ...), this at the same time allows for the investigation of the physical implications of the application of the theory's principles to new domains, such as Einstein (1905a) does for the electromagnetic field and Planck (1908a) for thermal radiation. A second reason for choosing the heuristics vs. normativity distinction over the dynamics vs. kinematics distinction is that using the second would suggest that my primary aim would be to pick a position in the existing debate within the philosophy of physics on the explanatory power of special relativity, whereas my concern is more with the history underlying the debate. 
Brown, H. (2005). Physical Relativity: Space-time structure from a dynamical perspective. Oxford University Press.

Bucherer, A. (1904). Mathematische Einführung in die Elektronentheorie. Teubner.

Bucherer, A. (1908). Messungen an Becquerelstrahlen. Die experimentelle Bestätigung der Lorentz-Einsteinschen Theorie. Physikalische Zeitschrift, $9(22): 755-762$.

Cohn, E. (1901). Über die Gleichungen des Elektromagnetischen Feldes für bewegte Körper. Annalen der Physik, 1: 29 - 56.

Corry, L. (1997). Hermann Minkowski and the Postulate of Relativity. Archive for History of Exact Sciences, 51(4): 273 - 314.

Cushing, J. T. (1981). Electromagnetic mass, relativity, and the Kaufmann experiments. American Journal of Physics, 49: 1133 - 1149.

Darrigol, O. (2001). The Historians' Disagreement over the Meaning of Planck's Quantum. Centaurus, 43: 219 - 239.

Ehrenfest, P. (1905). Über die physikalischen Voraussetzung der Planck'schen Theorie der irreversiblen Strahlungsvorgänge. Sitzungsberichte der Kaiserliche Akademie der Wissenschaft in Wien, MathematischNaturwissenschaftliche Klasse, 114: 1301 - 1314.

Ehrenfest, P. (1906). Zur Stabilitätsfrage bei den Bucherer-LangevinElektronen. Physikalische Zeitschrift, 7: 302 - 303. 
Ehrenfest, P. (1907). Die Translation deformierbarer Elektronen und der Flächensatz. Annalen der Physik, 23: 204 - 205.

Ehrenfest, P. (1909). Gleichförmige Rotation starrer Körper und Relativitätstheorie. Physikalische Zeitschrift, 10: 918.

Einstein, A. (1905a). Zur Elektrodynamik bewegter Körper. Annalen der Physik, 17: $891-921$.

Einstein, A. (1905b). Ist die Trägheit eines Körpers von seinem Energieinhalt abhängig? Annalen der Physik, 18: 639 - 641.

Einstein, A. (1905c). Über einen die Erzeugung und Verwandlung des Lichtes betreffenden heuristischen Gesichtspunkt. Annalen der Physik, 17: 132 148.

Einstein, A. (1906a). Das Prinzip von der Erhaltung des Schwerpunktsbewegung und die Trägheit der Energie. Annalen der Physik, 20: 627 - 633.

Einstein, A. (1906b). Zur Theorie der Lichterzeugung und Lichtabsorption. Annalen der Physik, 20: 199 - 206.

Einstein, A. (1906c). Über eine Methode zur Bestimmung des Verhältnisses der transversalen und longitudinalen Masse des Elektrons. Annalen der Physik, 21: $583-586$.

Einstein, A. (1907a). Bemerkungen zu der Notiz von Hrn. Paul Ehrenfest: "Die Translation deformierbarer Elektronen und der Flächensatz". Annalen der Physik, 23: 206 - 208. 
Einstein, A. (1907b). Über die vom Relativitätsprinzip geforderte Trägheit der Energie. Annalen der Physik, 23: 371 - 384.

Einstein, A. (1907c). Über das Relativitätsprinzip und die aus demselben gezogenen Folgerungen. Jahrbuch der Radioaktivität und Elektronik, 4: 411 $-462$.

Einstein, A. (1907d). Die Plancksche Theorie der Strahlung und die Theorie der spezifischen Wärme. Annalen der Physik, 22: 180 - 190.

Einstein, A. (1907e). Über die Möglichkeit einer neuen Prüfung des Relativitätsprinzip. Annalen der Physik, 23: 197 - 198.

Einstein, A. (1907f). Über die Gültigkeitsgrenze des Satzes vom thermodynamischen Gleichgewicht und über die Möglichkeit einer neuen Bestimmung der Elementarquanta. Annalen der Physik, 22: 596 - 572.

Einstein, A. \& Laub, J. (1908a). Über die elektromagnetische Grundgleichungen für bewegte Körper. Annalen der Physik, 26: 532 - 540 .

Einstein, A. \& Laub, J. (1908b). Über die im elektromagnetischen Felde auf ruhende Körper ausgeübten ponderomotorischen Kräfte. Annalen der Physik, 26: $541-550$.

Einstein, A. (1909a). Zum gegenwärtigen Stand des Strahlungsproblem. Physikalische Zeitschrift, 10: 185 - 193.

Einstein, A. (1909b). Über die Entwicklung unserer Anschauungen über das Wesen und die Konstitution der Strahlung. Physikalische Zeitschrift, 10: $817-825$. 
Einstein, A. (1917). Über die spezielle und allgemeine Relativitätstheorie. Braunschweig.

Gearheart, C. (2002). Planck, the Quantum, and the Historians. Physics in Perspective, 4: $170-215$.

Goldberg, S. (1970). The Abraham Theory of the Electron: The Symbiosis of Experiment and Theory. Archive for history of exact sciences, 7(1): 7 $-25$.

Goldberg, S. (1976). Max Planck's Philosophy of Nature and His Elaboration of the Special Theory of Relativity. Historical Studies in the Physical Sciences, 7: $125-160$.

Helmholtz, Hermann von. (1895). Ueber die physikalische Bedeutung des Princips der kleinsten Wirkung. In: Wissenschaftliche Abhandlungen III: $203-248$.

Hon, G. (1995). Is the Identification of an Experimental Error Contextually Dependent? The Case of Kaufmann's Experiments and its Varied Reception. In: Buchwald, J. Z. (Ed.): Scientific Practice: Theories and Stories of Doing Physics: $170-223$.

Hupka, K. E. (1909). Beitrag zur Kenntnis der trägen Masse bewegter Elektronen. Annalen der Physik, 31: 169 - 204.

Hupka, K. E. (1910). Zur Frage der trägen Masse bewegter Elektronen. Annalen der Physik, 33: 400 - 402. 
Jammer, M. (1961). Concepts of Mass in classical and modern physics. Harper Torchbooks.

Janssen, M. \& Mecklenburg, M. (2006). From classical to relativistic mechanics: electromagnetic models of the electron. In Hendricks, V. F. Jørgensen, K. F. Lützen, J. and Pedersen, S. A. (Eds.): Interactions: Mathematics, Physics and Philosophy, 1860 - 1930: 65 - 134.

Janssen, M. (2009). Drawing the Line between Kinematics and Dynamics in Special Relativity. Studies in History and Philosophy of Modern Physics, 40: $26-52$.

Jungnickel, C. \& McCormmach, R. (1990). Intellectual Mastery of Nature: Theoretical Physics from Ohm to Einstein. Volume 2 The Now Mighty Theoretical Physics 1870 - 1925. The University of Chicago Press.

Katzir, S. (2005). On "the electromagnetic world-view": A comment on an article by Suman Seth. Historical Studies in the Physical and Biological Sciences, 36(1): $189-192$.

Kaufmann, W. (1901a). Die magnetische und elektrische Ablenkbarkeit der Becquerelstrahlen und die scheinbare Masse der Elektronen. Nachrichten von der Gesellschaft der Wissenschaften zu Göttingen, MathematischPhysikalische Klasse: 143 - 155.

Kaufmann, W. (1901b). Die Entwicklung des Elektronenbegriffs. Physikalische Zeitschrift, 3: 9 - 15. 
Kaufmann, W. (1902). Über die elektromagnetische Masse des Elektrons. Nachrichten von der Gesellschaft der Wissenschaften zu Göttingen, Mathematisch-Physikalische Klasse: 291 - 296.

Kaufmann, W. (1903). Über die "Elektromagnetische Masse" der Elektronen. Nachrichten von der Gesellschaft der Wissenschaften zu Göttingen, Mathematisch-Physikalische Klasse: 90 - 103.

Kaufmann, W. (1906). Über die Konstitution des Elektrons. Annalen der Physik, 19: $487-553$.

Kaufmann, W. (1907). Bemerkungen zu Herrn Plancks: 'Nachtrag zu der Besprechung der Kaufmannschen Ablenkungsmessungen'. Deutsche Physikalische Gesellschaft. Verhandlungen, 9: $667-673$.

Kaufmann, W. (1908). Erwiderung an Herrn Stark. Deutsche Physikalische Gesellschaft. Verhandlungen, 10: $91-95$.

Klein, M. J. (1962). Max Planck and the Beginnings of the Quantum Theory. Archive for the history of the exact sciences, 1: $459-479$.

Klein, M. J. (1979). Einstein and the Development of Quantum Physics. In: French, A. (Ed.). Einstein: A Centenary Volume. Heinemann.

Klein, M. J. et al. (1993). The Collected Papers of Albert Einstein, Volume 5, The Swiss Years: Correspondence, 1902 - 1914. Princeton University Press.

Kragh, H. (1999). Quantum Generations: A History of Physics in the Twentieth Century. Princeton University Press. 
Kuhn, T. S. (1978). Black-Body Theory and the Quantum Discontinuity 1894 - 1912. Oxford University Press.

Laub, J. (1910). Über die experimentellen Grundlagen des Relativitätsprinzip. Jahrbuch der Radioaktivität und Elektronik, 7: 405 - 463.

Laue, M. (1911a). Zur Dynamik der Relativitätstheorie. Annalen der Physik, 35: $524-542$.

Laue, M. (1911b). Das Relativitätsprinzip. Braunschweig.

Levi-Civita, T. (1907). Sur le mouvement de l'électricité sans liaisons ni forces extérieures. Académie des sciences (Paris), Comptes Rendus, 145: 417 420.

Lorentz, H. A. (1895). Versuch einer Theorie der electrischen und optischen Erscheinungen in bewegten Körpern. Brill.

Lorentz, H. A. (1899). Simplified Theory of Electrical and Optical Phenomena in Moving Systems. Koninklijke Akademie van Wetenschappen te Amsterdam, Section of Sciences, Proceedings, 1: 427 - 442.

Lorentz, H. A. (1904). Electromagnetic phenomena in a system moving with any velocity smaller than that of light. English reprint of Koninklijke Akademie van Wetenschappen te Amsterdam, Section of sciences, Proceedings, 6, in Einstein, A. et al. (1952). The Principle of Relativity: $11-$ 34. Dover.

McCormmach, R. (1970). Einstein, Lorentz, and the Electron Theory. Historical Studies in the Physical Sciences, 2: $41-87$. 
Miller, A. I. (1981). Albert Einstein's Special Theory of Relativity: Emergence (1905) and Early Interpretation (1905 - 1911). Addison-Wesley Publishing Company.

Minkowski, H. (1907). Das Relativitätsprinzip. Annalen der Physik, [1915] 352: $927-938$.

Minkowski, H. (1908). Die Grundgleichungen für die elektromagnetischen Vorgänge in bewegten Körpern. Nachrichten von der Gesellschaft der Wissenschaften zu Göttingen, Mathematisch-Physikalische Klasse: 53 - 111.

Minkowski, H. (1909). Raum und Zeit. Jahresberichte der Deutschen Mathematiker-Vereinigung: $75-88$.

Planck, M. (1901a). Über das Gesetz der Energieverteilung im Normalspectrum. Annalen der Physik, 4: 553 - 563.

Planck, M. (1901b). Über die Elementarquanta der Materie und der Elektricität. Annalen der Physik, 4: $564-566$.

Planck, M. (1906a). Das Prinzip der Relativität und die Grundgleichungen der Mechanik. Deutsche Physikalische Gesellschaft. Verhandlungen, 8: 136 $-141$.

Planck, M. (1906b). Die Kaufmannschen Messungen der Ablenkbarkeit der $\beta$-Strahlen in ihrer Bedeutung für die Dynamik der Elektron. Physikalische Zeitschrift, 7: $753-761$.

Planck, M. (1907). Nachtrag zu der Besprechung der Kaufmannschen 
Ablenkungsmessungen. Verhandlungen Deutsche Physikalische Gesellschaft, 9(14): $301-305$.

Planck, M. (1908a). Zur Dynamik bewegter Systeme. Annalen der Physik, 26: $1-34$.

Planck, M. (1908b). Bemerkungen zum Prinzip der Aktion und Reaktion in der allgemeinen Dynamik. Physikalische Zeitschrift, 9(23): 828 - 830 .

Poincaré, H. (1905). Sur la dynamique de l'électron. Comptes Rendus de l'Academie des Sciences, CXL: $567-580$.

Poincaré, H. (1906). Sur la dynamique de l'électron. Rendiconti del Circolo Matematico di Palermo, 21: 129 - 175.

Pyenson, L. (1985). The Young Einstein: The Advent of Relativity. Taylor \& Francis.

Searle, G. F. C. (1897). On the steady motion of an electrified ellipsoid. Philosophical Magazine, 44: 329 - 341.

Seth, S. (2004). Quantum theory and the electromagnetic world-view. Historical Studies in the Physical and Biological Sciences, 35(1): 67 - 93.

Seth, S. (2005). Response to Shaul Katzir: "On the electromagnetic worldview". Historical Studies in the Physical and Biological Sciences, 36(1): $193-196$.

Seth, S. (2010). Crafting the Quantum: Arnold Sommerfeld and the Practice of Theory, 1890 - 1926. University of Chicago Press. 
Stachel, J. et al. (1989). The Collected Papers of Albert Einstein, Volume 2, The Swiss Years: Writings, 1900 - 1909. Princeton University Press.

Stark, J. (1906). Über die Lichtemission der Kanalstrahlen in Wasserstof. Annalen der Physik, 21: $401-456$.

Stark, J. (1907). Beziehung des Doppler-Effektes bei Kanalstrahlen zur Planckschen Strahlungstheorie. Physikalische Zeitschrift, 25: 913 - 919.

Stark, J. (1908). Bemerkung zu Herrn Kaufmanns Antwort auf einen Einwand von Herrn Planck. Deutsche Physikalische Gesellschaft, 10: 14 16.

Staley, R. (2008). Einstein's Generation: The Origins of the Relativity Revolution. University of Chicago Press.

Wien, W. (1900). Über die Möglichkeit einer elektromagnetischen Begründung der Mechanik. Annalen der Physik, 5: 501 - 513.

Wilson, H. A. (1904). On the Electric Effect of Rotating a Dielectric in a Magnetic Field. Royal Society of London, Philosophical Transactions A, 204: $121-137$.

Zahar, E. (1989). Einstein's Revolution: A Study in Heuristic. Open Court Publishing Company. 